\title{
TAME STACKS IN POSITIVE CHARACTERISTIC
}

\author{
DAN ABRAMOVICH, MARTIN OLSSON, AND ANGELO VISTOLI
}

\section{INTRODUCTION}

Since their introduction in [8, 4, algebraic stacks have been a key tool in the algebraic theory of moduli. In characteristic 0 , one often is able to work with Deligne-Mumford stacks, which, especially in characteristic 0, enjoy a number of nice properties making them almost as easy to handle as algebraic spaces. In particular, if $\mathcal{M}$ is a Deligne-Mumford stack and $M$ its coarse moduli space, then étale locally over $M$ we can present $\mathcal{M}$ as the quotient of a scheme by a finite group action. In characteristic 0 the formation of $M$ commutes with arbitrary base change, and if $\mathcal{M} \rightarrow S$ is flat then $M \rightarrow S$ is flat as well. A key property in characteristic 0 is that the pushforward functor $\mathrm{QCoh}(\mathcal{M}) \rightarrow \mathrm{QCoh}(M)$ is exact.

In characteristics $p>0$ the situation is not as simple. First, in many situations one needs to consider algebraic stacks (in the sense of Artin) with finite but possibly ramified diagonal. Examples include K3 surfaces, surfaces of general type, polarized torsors for abelian varieties, stable maps. Second, even Deligne-Mumford stacks may fail to have some desired properties, such as flatness of moduli spaces, as soon as the orders of stabilizers are divisible by the characteristics.

In this paper we isolate a class of algebraic stacks in positive and mixed characteristics, called tame stacks, which is a good analogue to the class of Deligne-Mumford stacks in characteristic 0, and arguably better than the class of Deligne-Mumford stacks in positive and mixed characteristics. Their defining property is precisely the key property described above: an algebraic stack with finite diagonal is tame if and only if the pushforward functor $\mathrm{QCoh}(\mathcal{M}) \rightarrow \mathrm{QCoh}(M)$ is exact (see Definition 3.1 and discussion therein for the precise hypotheses).

Our main theorem on tame stacks is Theorem 3.2. In particular, we show that an algebraic stack $\mathcal{M}$ is tame if and only if, locally in the étale topology of the coarse moduli space $M$, it can be presented as the quotient $[U / G]$ of a scheme by the action of a finite flat linearly reductive group scheme. Other desirable properties, such as flatness of coarse moduli spaces, commutation

Date: March 11, 2007.

Vistoli supported in part by the PRIN Project "Geometria sulle varietà algebriche", financed by MIUR. Olsson partially supported by NSF grant DMS-0555827 and an Alfred P. Sloan fellowship. Abramovich support in part by NSF grants DMS-0301695 and DMS0603284 . 
of the formation of moduli space with arbitrary base change, and stability of the tame property under pullbacks follow as corollaries.

It should be noted that it is significantly easier to show such a presentation as a quotient locally in the fppf topology. But the presentation in the étale topology is extremely useful for the applications we envision. This is one of the most intricate technical points in the paper.

The proofs of our results on tame stacks necessitate a good classification and study of finite flat linearly reductive group schemes. To our surprise, we have not found a good reference on this subject, and therefore developed it here. Our main theorem on linearly reductive group schemes is Theorem 2.19. In particular it says that a finite flat group scheme $G \rightarrow S$ is linearly reductive if and only if its geometric fibers $G_{x}$ are extensions $1 \rightarrow \Delta \rightarrow G_{x} \rightarrow$ $Q \rightarrow 1$ with tame and étale quotient $Q$ and diagonalizable kernel $\Delta$. While this classification is pleasing in its simplicity, it is also a bit disappointing, as it shows that the only finite groups admitting a linearly reductive reduction to characteristic $p>0$ have a normal and abelian $p$-Sylow subgroup.

Finite flat linearly reductive group schemes and their classification are the subject of Section 2 ,

In Section 3 we define the notion of tame stack, and prove the key local structure theorem (Theorem 3.2).

In a sequel to this paper, we develop the theory of twisted curves and twisted stable maps with a tame target, in analogy to [1]. This is for us the main motivation for the introduction of tame stacks.

In Appendix $\mathrm{A}$ we discuss rigidification of stacks. Discussion of rigidification has appeared in several places in the literature already, but unfortunately not in sufficient generality for the applications we have in mind. So we treat the most general case here.

Acknowledgments. We would like to thank Andrew Kresch, Frans Oort and René Schoof for useful discussions.

\section{Linearly REDUCTIVE FINITE GROUP SCHEMES}

Throughout the paper all schemes are assumed to be quasi-separated. (Recall that a scheme $S$ is quasi-separated when the diagonal $S \rightarrow S \times S$ is quasi-compact.)

In this section 2 all group schemes will be flat, finite and finitely presented over an arbitrary scheme. Such a group scheme $G \rightarrow S$ will be called constant if $G$ is the product of $S$ by a finite group.

2.1. Equivariant sheaves. Let $\pi: G \rightarrow S$ be a group scheme. A $G$ equivariant sheaf on $S$ is a sheaf with an action of $G$. There are (at least) four ways of defining an action of $G$ on a quasi-coherent sheaf.

(a) A quasi-coherent sheaf $F$ on $S$ extends naturally to a functor

$$
F:(\mathrm{Sch} / S)^{\mathrm{op}} \longrightarrow(\mathrm{Grp}) \text {. }
$$


If $f: T \rightarrow S$ is a morphism of schemes, then we define $F(T)$ as $\left(f^{*} F\right)(T)$; each $F(T)$ has the structure of an $\mathcal{O}(T)$-module.

Then an action of $G$ on $F$ is an $\mathcal{O}$-linear action of the functor

$$
G:(\mathrm{Sch} / S)^{\mathrm{op}} \longrightarrow(\mathrm{Grp})
$$

on $F$. In other words, for each $T \rightarrow S$ we have an action of the group $G(T)$ on the $\mathcal{O}(T)$-module $F(T)$, and this action is functorial in $T \rightarrow S$.

(b) Same as above, but the action of $G(T)$ on $F(T)$ is only defined for flat locally finitely presented morphisms $T \rightarrow S$.

(c) We have a sheaf of commutative Hopf algebras $\pi_{*} \mathcal{O}_{G}$ on $S$. Then an action of $G$ on a quasi-coherent sheaf $F$ is defined as a coaction $F \rightarrow$ $F \otimes \mathcal{O}_{S} \pi_{*} \mathcal{O}_{G}$ of this sheaf on $F$. Equivalently, in terms of the dual Hopf algebra $\mathbf{H}_{G}=\left(\pi_{*} \mathcal{O}_{G}\right)^{\vee}$, the "convolution hyperalgebra of $G$ ", it is an action $F \otimes \mathbf{H}_{G} \rightarrow F$.

(d) An action of $G$ on $F$ can also be defined as a isomorphism of $\mathcal{O}_{G}$-modules $\phi: \pi^{*} F \simeq \pi^{*} F$ satisfying the cocycle condition

$$
m^{*} \phi=\operatorname{pr}_{1}^{*} \phi \circ \operatorname{pr}_{2}^{*} \phi: \Pi^{*} F \simeq \Pi^{*} F
$$

where $m: G \times{ }_{S} G \rightarrow G$ is the multiplication morphism and $\Pi: G \times{ }_{S} G \rightarrow$ $S$ is the structure morphism.

The connections among these various definitions are as follows. The equivalence between (a) and (dd) is a particular case of [32, Proposition 3.49]. The same Proposition, applied to the fibered category whose object are pairs $(T \rightarrow S, F)$, where $T \rightarrow S$ is a flat finitely presented morphism, gives the equivalence between (IC) and (d). Finally, assigning a homomorphism of $\mathcal{O}_{G}$-modules $\phi: \pi^{*} F \simeq \pi^{*} F$ is equivalent to assigning a homomorphism of $\mathcal{O}_{S}$-modules $F \rightarrow \pi_{*} \pi^{*} F=F \otimes_{\mathcal{O}_{S}} \pi_{*} \mathcal{O}_{G}$; and using [32, Proposition 3.48 and Proposition 3.49] it is easy to see that $\phi$ satisfies the cocycle condition of (dd) if and only the corresponding homomorphism $F \rightarrow F \otimes_{\mathcal{O}_{S}} \pi_{*} \mathcal{O}_{G}$ is a coaction.

Using any of the definitions above of a $G$-action, there is an obvious definition of an equivariant homomorphism of quasi-coherent sheaves with a $G$-action. We will denote by $\mathrm{QCoh}(S)$ the category of quasi-coherent sheaves on $S$, and by $\mathrm{QCoh}^{G}(S)$ the category of $G$-equivariant quasi-coherent sheaves over $S$.

When $S$ is locally noetherian, we also denote by $\operatorname{Coh}(S)$ and $\operatorname{Coh}^{G}(S)$ the categories of coherent sheaves, respectively without and with a $G$-action.

The category $\mathrm{QCoh}^{G}(S)$ is also equivalent to the category $\mathrm{QCoh}\left(\mathcal{B}_{S} G\right)$ of quasi-coherent sheaves over the classifying stack $\mathcal{B}_{S} G$. We will define this in a way that is slightly different from, but equivalent to, the standard one ([20, Definition 13.2.2]). A quasi-coherent sheaf $F$ on $\mathcal{B}_{S} G$ of $\operatorname{QCoh}^{G}(S)$ associates with each $G$-torsor $P \rightarrow T$ an $\mathcal{O}(T)$-module $F(P \rightarrow T)$; also, for 
each commutative diagram

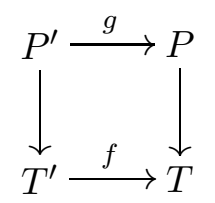

where the columns are $G$-torsors and $g$ is $G$-equivariant, we have a homomorphism $F(P \rightarrow T) \rightarrow F\left(P^{\prime} \rightarrow T^{\prime}\right)$ that is linear with respect to the natural ring homomorphism $\mathcal{O}(T) \rightarrow \mathcal{O}\left(T^{\prime}\right)$. These data are required to satisfy the following conditions.

(i) Suppose that we are given a $G$-torsor $P \rightarrow T$. Then we get a presheaf of $\mathcal{O}_{S}$-modules $F_{P \rightarrow T}$ defined by sending a Zariski-open subscheme $U$ to the $\mathcal{O}(U)$-module $F\left(\left.P\right|_{U} \rightarrow U\right)$. We require this to be a quasi-coherent sheaf on $S$.

(ii) Suppose that we have a commutative diagram like (2.1.1). Then we get a homomorphism of quasi-coherent sheaves

$$
F_{P \rightarrow T} \longrightarrow f_{*} F_{P^{\prime} \rightarrow T^{\prime}},
$$

defined by the given homomorphism

$$
\begin{gathered}
F_{P \rightarrow T}(U) \underset{\|}{F_{P^{\prime} \rightarrow T^{\prime}}}\left(f^{-1}(U)\right) \\
F\left(\left.P\right|_{U} \rightarrow U\right) \longrightarrow F\left(\left.P^{\prime}\right|_{f^{-1}(U)} \rightarrow f^{-1}(U)\right)
\end{gathered}
$$

for each open subscheme $U \subseteq T$. Then the corresponding homomorphism $f^{*} F_{P \rightarrow T} \rightarrow F_{P^{\prime} \rightarrow T^{\prime}}$ is required to be an isomorphism.

There is an obvious notion of homomorphism of quasi-coherent sheaves on $\mathcal{B}_{S} G$ : such a homomorphism $\phi: F \rightarrow F^{\prime}$ assigns to every $G$-torsor $P \rightarrow T$ a homomorphism of $\mathcal{O}(T)$-modules $\phi_{P \rightarrow T} F(P \rightarrow T) \rightarrow F^{\prime}(P \rightarrow T)$, in such a way that given any commutative diagram (2.1.1), the diagram

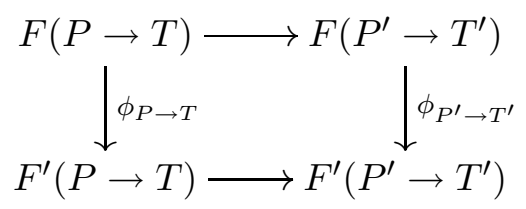

commutes. The category $\mathrm{Q} \operatorname{Coh}\left(\mathcal{B}_{S} G\right)$ is the category of quasi-coherent sheaves on $\mathcal{B}_{S} G$, the arrows being homomorphisms.

The equivalence between $\mathrm{QCoh}\left(\mathcal{B}_{S} G\right)$ and $\mathrm{QCoh}^{G}(S)$ is as follows.

Suppose that $F$ is an object of $\mathrm{QCoh}^{G}(S)$. If $T$ is an $S$-scheme and $P \rightarrow T$ is a $G$-torsor, and $h: P \rightarrow S$ is the composite of the given morphisms $P \rightarrow T$ and $T \rightarrow S$, then the pullback $h^{*} F$ is a quasi-coherent sheaf over $P$ with a $G$-action. On the other hand, by descent theory we have an equivalence between the category of $G$-equivariant quasi-coherent sheaves on $P$ and the category of quasi-coherent sheaves on $T$ ([32, Theorem 4.46]); we define 
$\Phi(F)_{P \rightarrow T}$ to be a quasi-coherent sheaf on $T$ whose pullback to $P$ is isomorphic to $h^{*} F$ a $G$-equivariant sheaf. It is easy to see the function that sends $P \rightarrow T$ into $\Phi(F)_{P \rightarrow T}$ has a natural structure of a quasi-coherent sheaf on $\mathcal{B}_{S} G$, and that a homomorphism $f: F \rightarrow F^{\prime}$ of $G$-equivariant quasi-coherent sheaves on $S$ induces a homomorphism $\phi(f): \Phi F \rightarrow \Phi F^{\prime}$ of quasi-coherent sheaves on $\mathcal{B}_{S} G$. This defines the functor $\Phi: \mathrm{QCoh}^{G}(S) \rightarrow \mathrm{QCoh}\left(\mathcal{B}_{S} G\right)$.

Let us define the inverse functor $\Psi: \mathrm{QCoh}\left(\mathcal{B}_{S} G\right) \rightarrow \mathrm{QCoh}^{G}(S)$. Given a quasi-coherent sheaf $F$ on $\mathcal{B}_{S} G$, we define the quasi-coherent sheaf $\Psi F$ on $S$ to be the sheaf $F_{G \rightarrow S}$ associated with the trivial $G$-torsor $G \rightarrow S$. For each morphism $f: T \rightarrow S$, the action of $G(T)$ on $f^{*} \Psi F$ is defined as follows: an element of $G(T)$ induces an an automorphism of the $G$-torsor $G_{T} \rightarrow T$, which in turns induces an automorphism of $F_{G_{T} \rightarrow T} \simeq f^{*} \Psi F$. It is easy to see that $\Psi$ extends naturally to a functor $\Psi: \operatorname{QCoh}\left(\mathcal{B}_{S} G\right) \rightarrow \mathrm{QCoh}^{G}(S)$.

It is easy to see that the composite $\Phi \Psi$ is isomorphic to $\mathrm{id}_{\mathrm{QCoh}^{G}(S)}$. It is slightly less trivial to show that $\Psi \Phi$ is isomorphic to $\operatorname{id}_{\mathrm{QCoh}\left(\mathcal{B}_{S} G\right)}$. The point is the following. Given a quasi-coherent sheaf $F$ on $\mathcal{B}_{S} G$ and a $G$-torsor $\rho: P \rightarrow T$ on an $S$-scheme $f: T \rightarrow S$, the pullback $\operatorname{pr}_{2}: P \times_{T} P \rightarrow P$ of $P \rightarrow T$ to $P$ has canonical section: this induces a cartesian diagram

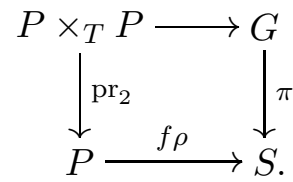

Since the pullback $\rho^{*} F_{P \rightarrow T}$ is isomorphic to $F_{P \times_{T} P \rightarrow P}$, this diagram induces an isomorphism of $\rho^{*} F_{P \rightarrow T}$ with $\rho^{*} f^{*} F_{G \rightarrow S}$. This isomorphism is easily seen to be $G_{T}$-equivariant; hence it descends to an isomorphism $F_{P \rightarrow T} \simeq$ $f^{*} F_{G \rightarrow S}=(\Psi \Phi) F$.

Suppose that $\phi: H \rightarrow G$ is a homomorphism of group schemes, there are two natural additive functors, the restriction functor

$$
\phi^{*}: \mathrm{QCoh}^{G}(S) \longrightarrow \mathrm{QCoh}^{H}(S)
$$

and the induction functor

$$
\phi_{*}: \mathrm{QCoh}^{H}(S) \longrightarrow \mathrm{QCoh}^{G}(S) .
$$

The first is evident. The second, $\phi_{*}$ can be defined using functorial actions, or using the convolution algebras: $\phi_{*} F=F \otimes_{\mathbf{H}_{H}} \mathbf{H}_{G}$. It is also useful to think about them as follows: $\phi$ induces a morphism of algebraic stacks

$$
\Phi: \mathcal{B}_{S} H \longrightarrow \mathcal{B}_{S} G
$$

defined as usual by sending a principal $H$-bundle $Q \rightarrow T$ to the principal $G$-bundle $\left(Q \times_{T} G_{T}\right) / H_{T}$, the quotient taken with $H_{T}$ acting via $(q, g) \mapsto$ $\left(q h, h^{-1} g\right)$. Then $\phi^{*}$ is pullback of quasi-coherent sheaves along $\Phi$, while $\phi_{*}$ is pushforward along $\Phi$.

Remark 2.2. A few important points about these functors: 
(1) The functor $\phi^{*}$ is always exact. Indeed, in terms of actions, $\phi^{*} F$ is the same sheaf $F$ but with the $G$ action replaced by the action of $H$ through $\phi$, and the action does not intervene in exactness.

(2) If $H$ is a subgroup scheme of $G$, then $\Phi$ is finite, and in particular affine; hence $\phi_{*}$ is exact. In this case we denote it by $\operatorname{Ind}_{H}^{G}$.

(3) If we think of the structure morphism $\pi: G \rightarrow S$ as a homomorphism to the trivial group scheme and $F$ is a $G$-equivariant quasi-coherent sheaf on $S$, then we denote $\pi_{*} F$ by $F^{G}$. This quasi-coherent sheaf $F^{G}$ is naturally embedded in $F$ (by the adjunction map $\pi^{*} \pi_{*} F \rightarrow F$ ), and is called the invariant subsheaf.

The invariant subsheaf can also be defined directly from any of the various definition of an action of $G$ on a quasi-coherent sheaf. For example, if $\alpha: F \rightarrow F \otimes \mathcal{O}_{S} \pi_{*} \mathcal{O}_{G}$ is a coaction, then $F^{G}$ is the kernel of $\alpha-\iota$, where $\iota: F \rightarrow F \otimes_{\mathcal{O}_{S}} \pi_{*} \mathcal{O}_{G}$ is the trivial coaction, given by $s \mapsto s \otimes 1$.

(4) Suppose $\phi: H \rightarrow G$ is surjective, with kernel a flat group scheme $K$. For $F \in \mathrm{QCoh}^{H}(S)$ we have $\phi_{*} F=F^{K}$ with the induced action of $G$. On the other hand if $F \in \mathrm{QCoh}^{G}(S)$ then the adjunction morphism $F \rightarrow \phi_{*} \phi^{*} F$ is an isomorphism, since the action of $K$ on $\phi^{*} F$ is trivial. In other words, we have a canonical isomorphism $\phi_{*} \circ \phi^{*} \simeq$ id.

\subsection{Linearly reductive group schemes.}

Definition 2.4. A group scheme $G \rightarrow S$ is linearly reductive if the functor $\mathrm{QCoh}^{G}(S) \rightarrow \mathrm{QCoh}(S)$ sending $F$ to $F^{G}$ is exact.

Proposition 2.5. Assume that $S$ is noetherian. Then $G$ is linearly reductive if and only if the functor $\operatorname{Coh}^{G}(S) \rightarrow \operatorname{Coh}(S)$ defined as $F \mapsto F^{G}$ is exact.

Proof. We need to show that given a surjection $F \rightarrow F^{\prime}$ of $G$-equivariant quasi-coherent sheaves on $S$, the induced morphism $F^{G} \rightarrow F^{\prime}{ }^{G}$ is also surjective. Since $S$ is noetherian, every quasi-coherent sheaf with an action of $G$ is a direct limit of coherent subsheaves with an action of $G$ (see, e.g. Lemma 2.1 31 or 29]). By replacing $F^{\prime}$ with an arbitrary coherent subsheaf and $F$ with its inverse image in $F$, we may assume that $F^{\prime}$ is coherent.

Let $\left\{F_{i}\right\}$ the inductive system of coherent $G$-equivariant subsheaves of $F$ : since $S$ is noetherian and $F^{\prime}$ is coherent, there will exists some $i$ such that $F_{i}$ surjects onto $F^{\prime}$. Then $F_{i}^{G} \rightarrow F^{\prime G}$ is surjective, so $F^{G} \rightarrow F^{\prime G}$ is surjective.

In particular, if $k$ is a field, the category of coherent sheaves on Spec $k$ with an action of $G$ is equivalent to the category of finite-dimensional representations of $G$; hence a finite group scheme over a field is linearly reductive if and only if the functor $V \mapsto V^{G}$, from finite-dimensional representations of $G$ to vector spaces, is exact.

Another, perhaps more customary, way to state this condition is to require that every finite-dimensional representation of $G$ be a sum of irreducible representations. 
Proposition 2.6. Let $S^{\prime} \rightarrow S$ be a morphism of schemes, $G \rightarrow S$ a group scheme, $G^{\prime} \stackrel{\text { def }}{=} S^{\prime} \times{ }_{S} G$.

(a) If $G \rightarrow S$ is linearly reductive, then $G^{\prime} \rightarrow S^{\prime}$ is linearly reductive.

(b) If $G^{\prime} \rightarrow S^{\prime}$ is linearly reductive and $S^{\prime} \rightarrow S$ is flat and surjective, then $G \rightarrow S$ is linearly reductive.

Proof. Let us prove part (b). There is a cartesian diagram

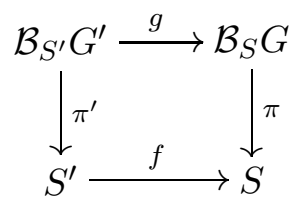

from which we deduce that the two functors $f^{*} \pi_{*}$ and $\pi_{*}^{\prime} g^{*}$ are isomorphic. Since $f$ is flat, $g$ is flat as well; also $\pi_{*}^{\prime}$ is exact by assumption, so $\pi_{*}^{\prime} g^{*}$ is exact, hence $f^{*} \pi_{*}$ is exact. But since $f$ is faithfully flat we have that $\pi_{*}$ is exact, as required.

Now for part (回).

First assume that $S^{\prime}$ is an affine open subscheme of $S$. Then the embedding $j: S^{\prime} \hookrightarrow S$ is quasi-compact, since $S$ is quasi-separated: hence the pushforward $j_{*}$ takes quasi-coherent sheaves into quasi-coherent sheaves ([10, I, Corollaire 9.2.2]). It is easy to see that if $F^{\prime}$ is a $G_{S^{\prime}}$-equivariant quasi-coherent sheaf on $S^{\prime}$, the action of $G_{S^{\prime}}$ on $F^{\prime}$ extends to an action of $G$ on $j_{*} F^{\prime}$ : if $T \rightarrow S$ is a flat morphism and $T^{\prime}$ is the inverse image of $S^{\prime}$ in $T$, then $G\left(T^{\prime}\right)$ acts on $F\left(T^{\prime}\right)=j_{*} F(T)$, and this induces an action of $G(T)$ on $j_{*} F(T)$ via the restriction homomorphism $G(T) \rightarrow G\left(T^{\prime}\right)$. Then every exact sequence

$$
0 \longrightarrow F_{1}^{\prime} \longrightarrow F_{2}^{\prime} \longrightarrow F_{3}^{\prime} \longrightarrow 0
$$

of $G$-equivariant quasi-coherent sheaves on $S^{\prime}$ extends to an exact sequence

$$
0 \longrightarrow F_{1} \longrightarrow F_{2} \longrightarrow F_{3} \longrightarrow 0
$$

of $G$-equivariant quasi-coherent sheaves on $S$ : we take $F_{1} \stackrel{\text { def }}{=} j_{*} F_{1}^{\prime}$ and $F_{2} \stackrel{\text { def }}{=} j_{*} F_{2}^{\prime}$, while $F_{3}$ is defined to be the image of $j_{*} F_{2}^{\prime}$ in $j_{*} F_{3}^{\prime}$. Since taking invariants commutes with restriction to open subschemes, the result follows.

If $\left\{S_{i}\right\}$ is an open covering of $S$ by affines, and each restriction $G_{S_{i}}$ is linearly reductive over $S_{i}$, then the disjoint union $\sqcup_{i} G_{S_{i}}$ is linearly reductive over $\sqcup_{i} S_{i}$; we conclude from part (b) that $G$ is linearly reductive over $S$. Hence being linearly reductive is a local property in the Zariski topology. So to prove part (司) it suffices to show that when $S$ and $S^{\prime}$ are both affine the functor $\mathrm{QCoh}^{G^{\prime}}\left(S^{\prime}\right) \rightarrow \mathrm{QCoh}\left(S^{\prime}\right)$ is exact.

Then $g$ is also affine, so the functor $g_{*}: \mathrm{QCoh}^{G^{\prime}}\left(S^{\prime}\right) \rightarrow \mathrm{QCoh}^{G}(S)$ is exact. By assumption $\pi_{*}$ is exact, therefore $\pi_{*} g_{*}=f_{*} \pi_{*}^{\prime}$ is exact. But the functor $f_{*}$ has the property that a sequence $F_{1} \rightarrow F_{2} \rightarrow F_{3}$ is exact if and only if $f_{*} F_{1} \rightarrow f_{*} F_{2} \rightarrow f_{*} F_{3}$ is exact. It follows that $\pi_{*}^{\prime}$ is exact, as required. 
Proposition 2.7. $\quad$ The class of linearly reductive group schemes is closed under taking

(a) subgroup schemes,

(b) quotients, and

(c) extensions.

Proof. For part (国), consider a subgroup scheme $G^{\prime} \subset G$ and the resulting commutative diagram

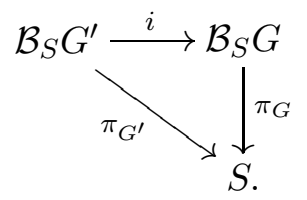

It is enough to observe that $i_{*}=\operatorname{Ind}_{G^{\prime}}^{G}$ is exact (Remark 2.2 (2) ). Since $\pi_{G *}$ is exact by assumption, and since $\pi_{G^{\prime} *} \simeq \pi_{G *} \circ i_{*}$ part (a) follows.

For parts (b) and ( $(\mathrm{C})$, consider an exact sequence

$$
1 \rightarrow G^{\prime} \rightarrow G \rightarrow G^{\prime \prime} \rightarrow 1
$$

and the corresponding commutative diagram

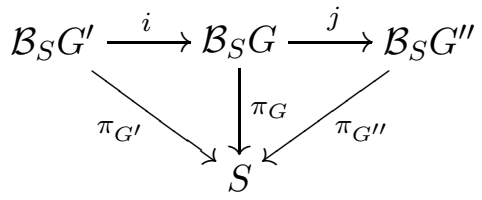

To prove part (b), suppose that $G$ is linearly reductive, so $\pi_{G *}$ is exact. Recall that $j^{*}$ is exact and $j_{*} \circ j^{*}$ is isomorphic to the identity, so

$$
\pi_{G^{\prime \prime} *} \simeq \pi_{G^{\prime \prime} *} \circ j_{*} \circ j^{*} \simeq \pi_{G *} \circ j^{*}
$$

is exact, as required.

For part (C), we have by assumption that $\pi_{G^{\prime} *}$ and $\pi_{G^{\prime \prime} *}$ are exact. Consider the cartesian diagram

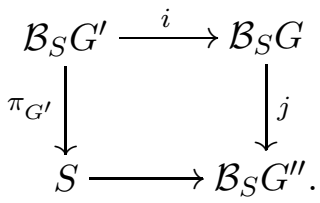

The formation of $j_{*}$ commutes with flat base change on $\mathcal{B}_{S} G^{\prime \prime}$ and $S \rightarrow \mathcal{B}_{S} G^{\prime \prime}$ is faithfully flat. Thus to verify that $j_{*}$ is exact it suffices to show that $\pi_{G^{\prime}}$ is exact, which holds since $G^{\prime}$ is assumed linearly reductive. Therefore $j_{*}$ is exact (concretely, taking invariants of a $G$ sheaf by $G^{\prime}$ is exact even if when consider the induced $G^{\prime \prime}$-action). So

$$
\pi_{G *}=\pi_{G^{\prime \prime} *} \circ j_{*}
$$

is exact, as required. 
2.8. Classifying linearly reductive group schemes. We will say that a finite group scheme $\Delta \rightarrow S$ is diagonalizable if it is abelian and its Cartier dual is constant. The standard definition only requires the Cartier dual to be constant on the connected components of $S$, (see [9, Exposé VII]), but the distinction is of little importance to us. We say that $\Delta \rightarrow S$ is locally diagonalizable if its Cartier dual is étale.

Given a finite group scheme $\Delta \rightarrow S$, the following conditions are clearly equivalent.

(a) $\Delta$ is locally diagonalizable.

(b) Locally in the étale topology, $\Delta$ is diagonalizable.

(c) Locally in the fppf topology, $\Delta$ is diagonalizable.

(d) Locally in the fpqc topology, $\Delta$ is diagonalizable.

A finite étale group scheme $H \rightarrow S$ is said to be tame if its degree is prime to all residue characteristics.

Definition 2.9. A group scheme $\pi: G \rightarrow S$ is well-split if it is isomorphic to a semidirect product $H \ltimes \Delta$, where $H$ is constant and tame and $\Delta$ is diagonalizable.

It is locally well-split if there is an fpqc cover $\left\{S_{i} \rightarrow S\right\}$, such that the group scheme $S_{i} \times{ }_{S} G \rightarrow S_{i}$ is well-split for each $i$.

In characteristic 0 every finite flat group scheme is étale and tame, hence locally constant, hence locally well-split.

Proposition 2.10. Every locally well-split group scheme is linearly reductive.

Proof. By Proposition [2.6 it suffices to consider well-split group schemes.

The Proposition holds for such group schemes by Proposition 2.7 (ㄷ), as it holds for diagonalizable group schemes (see [9, Exposé VII]), and tame constant group schemes (by Maschke's Lemma).

Lemma 2.11. Let $G$ be a locally well-split group scheme over a field $k$, let $\Delta_{0}$ be the connected component of the identity, and $H=G / \Delta_{0}$.

(a) The group scheme $\Delta_{0}$ is locally diagonalizable, and $H$ is étale and tame.

(b) There exists a finite purely inseparable extension $k^{\prime}$ of $k$, such that $G_{k^{\prime}}$ is a semidirect product $H_{k^{\prime}} \ltimes \Delta_{0 k^{\prime}}$. In particular, if $k$ is perfect, then $G$ is a semidirect product $H \ltimes \Delta_{0}$.

(c) There exists a finite extension $k^{\prime}$ of $k$ such that $G_{k^{\prime}}$ is well-split. In particular, if $k$ is algebraically closed, then $G$ is well-split.

Proof. For part (国), we may prove the statement after extending the base field, so we may assume that $G$ is well-split (note that since $\Delta_{0}$ is a connected scheme with a $k$-rational point, $\Delta_{0} \otimes_{k} k^{\prime}$ is connected for any field extension $k \rightarrow k^{\prime}$ ). Then $G$ contains a locally diagonalizable subgroup $\Delta$ such that $G / \Delta$ is étale; hence $\Delta_{0}$ coincides with the connected component in $\Delta$; hence it is diagonalizable, and the quotient $H=G / \Delta_{0}$ is étale and tame. 
For part (B), there is a finite purely inseparable extension $k^{\prime}$ of $k$ such that $\left(G_{k^{\prime}}\right)_{\text {red }}$ is smooth over $k^{\prime}$. Then $\left(G_{k^{\prime}}\right)_{\text {red }}$ is a subgroup scheme of $G$, and it maps isomorphically to $H_{k^{\prime}}$.

For part (ㄷ), because of part (b) we may assume that $G$ is of the form $H \ltimes \Delta_{0}$, where $H$ is étale and tame and $\Delta_{0}$ is locally diagonalizable. After passing to a finite separable extension of $k$, the group scheme $\Delta_{0}$ becomes diagonalizable and $H$ becomes constant.

Remark 2.12. In general, if $k$ is not perfect a non-trivial extension is necessary to obtain the splitting. For example suppose $k=k_{0}(a)$, where $a$ is an indeterminate and $k$ of characteristic $p>2$. Consider the semidirect product $\Gamma \stackrel{\text { def }}{=} \mathrm{C}_{2} \ltimes \boldsymbol{\mu}_{p}$ over $k$, where $\mathrm{C}_{2}$ is a cyclic group of order 2 , whose generator $s$ acts on $\boldsymbol{\mu}_{p}$ as $s \cdot t=t^{-1}$. Conjugation gives a right action of $\boldsymbol{\mu}_{p}$ on $\Gamma$; given the Kummer extension $k^{\prime}=k[t] /\left(t^{p}-a\right)$, take the quotient $G$ of $\Gamma \times{ }_{k}$ Spec $k^{\prime}$ by the diagonal action of $\boldsymbol{\mu}_{p}$. A simple calculation shows that $\boldsymbol{\mu}_{p}$ acts on the connected component $s \boldsymbol{\mu}_{p}$ of $\Gamma$ by the formula $y^{-1}(s x) y=s y^{2} x$.

Note that since the conjugation action of $\boldsymbol{\mu}_{p}$ on $\Gamma$ is by group homomorphisms, the diagonal action of $\boldsymbol{\mu}_{p}$ on $\Gamma \times \times_{\text {Spec } k}$ Spec $k^{\prime}$ defines descent data for the group scheme $\Gamma \times_{\text {Spec } k}$ Spec $k^{\prime}$ over Spec $k^{\prime}$, and therefore the quotient $G$ has a group scheme structure. This group scheme $G$ contains $\boldsymbol{\mu}_{p}$ as the connected component of the identity, and $G / \boldsymbol{\mu}_{p}$ is isomorphic to $\mathrm{C}_{2}$, but the other connected component is a copy of $\operatorname{Spec} k[t] /\left(t^{p}-a^{2}\right)$, whence the sequence is not split.

Proposition 2.13. Let $k$ be a field, $G \rightarrow$ Spec $k$ a finite group scheme. Then $G$ is linearly reductive if and only if it is locally well-split.

Proof. Let $\bar{k}$ be the algebraic closure of $k$; then by Proposition 2.6 the group scheme $G_{\bar{k}}$ is linearly reductive (respectively locally well-split) if and only if $G$ is linearly reductive (respectively locally well-split); so we may assume that $k$ is algebraically closed. We know that locally well-split groups are linearly reductive, so assume that $G$ is linearly reductive. Denote by $p$ the characteristic of $k$.

Let $G_{0}$ be the connected component of the identity in $G$. Then $G / G_{0}$ is a linearly reductive constant group. If it were not tame it would contain a subgroup of order $p$, which is not linearly reductive. So we may assume that $G$ is connected, and show that it is diagonalizable.

The following lemma may be known to the experts, but we have not found a reference.

Lemma 2.14. If a connected finite group scheme $G$ over an algebraically closed field contains a diagonalizable normal subgroup $H$, and $G / H$ is again diagonalizable, then $G$ is also diagonalizable.

Proof. If we show that $G$ is abelian, then it is diagonalizable: its Cartier dual is an extension with a constant quotient and a constant subgroup, which is therefore a constant group scheme, because the base field is algebraically closed. 
The action by conjugation of $G$ on $H$ defines a homomorphism of group schemes $G \rightarrow \underline{\mathrm{Aut}}_{\mathrm{Gr}-\mathrm{Sch} / k}(H)=\underline{\mathrm{Aut}}_{\mathrm{Gr}-\mathrm{Sch} / k}\left(H^{\mathrm{C}}\right)$, where $H^{\mathrm{C}}$ is the Cartier dual of $H$; but the domain is local, while the target is constant, so this homomorphism is trivial. Equivalently, $H$ is central in $G$.

Let $A$ be a commutative $k$-algebra. The groups $H(A)$ and $G(A) / H(A)$ are commutative, hence, by "calculus of commutators" (see [11], Section 6, in particular Lemma 6.1), we have a bilinear map

$$
\begin{aligned}
G(A) \times G(A) & \longrightarrow H(A) \\
(x, y) & \mapsto[x, y]
\end{aligned}
$$

This is functorial in $A$, therefore the commutator gives a bilinear map $G \times$ $G \rightarrow H$, and since $H$ is central this gives a bilinear map $Q \times Q \rightarrow H$, where we have set $Q \stackrel{\text { def }}{=} G / H$. In particular we get a map of sheaves $Q \rightarrow$ $\underline{\operatorname{Hom}}_{\text {Grp-Sch } / k}(Q, H)$, where both source and target are representable. But again the domain is local and the target is étale, hence $Q$ is mapped to the trivial map, in other words the commutator $Q \times Q \rightarrow H$ maps to the identity in $H$. This means that the commutator is trivial, hence $G$ is abelian.

So we may proceed by induction on the dimension of the vector space $\mathrm{H}^{0}\left(G, \mathcal{O}_{G}\right)$, and assume that $G$ does not contain any nontrivial normal subgroup schemes. In particular, the Frobenius kernel $G_{1}$ of $G$ is a normal subgroup scheme of $G$, which does not coincide with the identity, unless $G$ is trivial: so we have that $G=G_{1}$. In [22], p. 139 one says that $G$ has height 1 . Connected group schemes of height 1 are classified by their $p$-Lie algebras (see, e.g., [22], p. 139).

Lemma 2.15 (Jacobson 15, Chapter 5, Exercise 14, p. 196). Let $G$ be a non-abelian group scheme of height 1 . Then $G$ contains $\boldsymbol{\alpha}_{p}$, and hence is not linearly reductive.

Proof. Considering the $p$-lie algebra $\mathfrak{g}$ of $G$, we need to find an element $w \in \mathfrak{g}$ such that $w^{p}=0$. Since $\mathfrak{g}$ is finite dimensional, for each $v \in \mathfrak{g}$ there is a minimal $n$ such that $\left\{v, v^{p}, v^{p^{2}}, \ldots, v^{p^{n}}\right\}$ is linearly dependent, giving a monic $p$-polynomial

$$
f_{v}(x)=x^{p^{n}}+a_{n-1}^{(v)} x^{p^{n-1}}+\cdots+a_{0}^{(v)} x
$$

such that $f_{v}(v)=0$.

Note that if $a_{0}^{(v)}=0$ then the nonzero element

$$
w \stackrel{\text { def }}{=} f_{v}^{1 / p}(v)=v^{p^{n-1}}+\left(a_{n-1}^{(v)}\right)^{1 / p} v^{p^{n-2}}+\cdots+\left(a_{1}^{(v)}\right)^{1 / p} v
$$

satisfies $w^{p}=0$. So, arguing by contradiction, we may assume that $a_{0}^{(v)} \neq 0$ for all nonzero $v$, i.e. $f_{v}$ is separable. Since the minimal polynomial of $\operatorname{ad}(v)$ divides $f_{v}$, we have that $\operatorname{ad}(v)$ is semisimple for every nonzero $v$.

Since $\mathfrak{g}$ is assumed non-commutative, there is $v$ with $\operatorname{ad}(v) \neq 0$, hence it has a nonzero eigenvector $v^{\prime}$ with nonzero eigenvalue. But then the action of 
$\operatorname{ad}\left(v^{\prime}\right)$ on $\operatorname{Span}\left(v, v^{\prime}\right)$ is nonzero and nilpotent, contradicting semisimplicity.

Back to the proposition, we deduce that $G$ is abelian. Every subgroup scheme is normal, so $G$ cannot contain any proper subgroup scheme. But by Cartier duality the only local abelian group schemes with this property are $\boldsymbol{\alpha}_{p}$ and $\boldsymbol{\mu}_{p}$; and again $\boldsymbol{\alpha}_{p}$ is not linearly reductive. Hence $G=\boldsymbol{\mu}_{p}$, and we are done.

This completes our analysis for the case of group schemes over fields. To handle the general case we need the following fact.

Lemma 2.16. Let $G \rightarrow S$ be a finite flat group scheme of finite presentation. Assume that there is a point $s=\operatorname{Spec} k(s) \in S$ such that the fiber $G_{s} \rightarrow$ Spec $k(s)$ is locally well-split. Then there exists a flat quasi-finite map $U \rightarrow$ $S$ of finite presentation, whose image includes s, such that $G_{U}$ is well-split.

In particular, let $V$ be the image of $U$ in $S$, which is open; then the restriction $G_{V} \rightarrow V$ is locally well-split.

Proof. By standard arguments, we may assume that $S$ is connected, affine and of finite type over $\mathbb{Z}$. By Lemma 2.11 (ㄷ) , there is a finite extension $k$ of $k(s)$ such that $G_{k}$ is of the form $H \ltimes \Delta_{0}$, where $\Delta_{0}$ is a connected diagonalizable group scheme and $H$ is a constant group scheme, associated with a finite group $\Gamma$. After base change by a quasi-finite flat morphism over $S$, we may assume that $k(s)=k$. The group scheme $\Delta_{0}$ extends uniquely to a diagonalizable group scheme $\Delta_{0}$ on $S$, that we still denote by $\Delta_{0}$. Also, we denote again by $H$ the group scheme over $S$ associated with $\Gamma$; the action of $H$ on $\Delta_{0}$, which is defined over $s$, extends uniquely to an action of $H$ on $\Delta_{0}$. Set $G^{\prime}=H \ltimes \Delta_{0}$. We claim that $G$ and $G^{\prime}$ become isomorphic after passing to a flat morphism of finite type $U \rightarrow S$, whose image includes $s$. We use the following lemma, which shows that after passing to an étale neighborhood of $s$ in $S$ there exists a $\left(G, G^{\prime}\right)$-bitorsor $I \rightarrow S$. Given such a bitorsor, we have that $G$ is the group scheme of automorphisms of $I$ as a $G^{\prime}$-torsor, and is thus the twisted form of $G^{\prime}$ coming from $I$ and from the homomorphism $G^{\prime} \rightarrow \operatorname{Aut}\left(G^{\prime}\right)$ given by conjugation; so if $I \rightarrow S$ has a section, $G$ and $G^{\prime}$ are isomorphic. Hence the pullbacks of $G$ and $G^{\prime}$ to $I$ are isomorphic, and $I \rightarrow S$ is flat and quasi-finite. This completes the proof.

Lemma 2.17. Let $G \rightarrow S$ and $G^{\prime} \rightarrow S$ be two group schemes over $S$, and let $s \in S$ be a point such that the fibers $G_{s}$ and $G_{s}^{\prime}$ are linearly reductive and isomorphic. Then there exists an étale neighborhood $s \rightarrow U \rightarrow S$ of $s$ in $S$ and $a\left(G_{U}, G_{U}^{\prime}\right)$-bitorsor $I \rightarrow U$.

Proof. We can pass to the henselization $R$ of the local ring $\mathcal{O}_{S, s}$, and assume that $S=\operatorname{Spec} R$ and that $s$ is the closed point of $S$. Call $\mathfrak{m}$ the maximal ideal of $R$ and set $R_{n} \stackrel{\text { def }}{=} R / \mathfrak{m}^{n+1}$ and $S_{n} \stackrel{\text { def }}{=} \operatorname{Spec} R_{n}$. We will show that there exist a sequence of $\left(G, G^{\prime}\right)$-bitorsors $I_{n} \rightarrow S_{n}$, such that the restriction 
of each $I_{n}$ to $S_{n-1}$ is isomorphic to $I_{n-1}$; then the result follows from Artin's approximation theorem.

We present two methods of proof, one abstract and one more explicit. Both use deformation theory.

METHOD 1: RIGIDITY USING THE COTANGENT COMPLEX.

By [12, Remarque 1.6.7], a $\left(G, G^{\prime}\right)$-bitorsor $I_{n} \rightarrow S_{n}$ is the same as an equivalence of fibered categories $\rho_{n}: \mathcal{B}_{S_{n}} G_{S_{n}} \simeq \mathcal{B}_{S_{n}} G_{S_{n}}^{\prime}$. We inductively construct a compatible system of such isomorphisms. So suppose $\rho_{n}$ has been constructed. We then wish to find a dotted arrow filling in the diagram

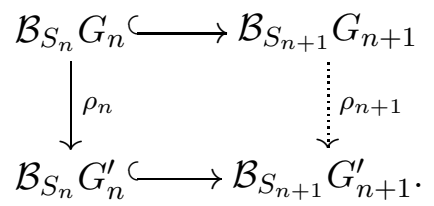

Note that any such morphism $\rho_{n+1}$ is automatically an isomorphism since $G_{n+1}$ and $G_{n+1}^{\prime}$ are flat over $S_{n+1}$. Let $\mathbf{L}_{\mathcal{B} G_{s}} \in D_{\text {coh }}\left(\mathcal{O}_{\mathcal{B} G_{s}}\right)$ denote the cotangent complex of $\mathcal{B} G_{s}$ over $s$ as defined in [20] (and corrected in [23]).

Lemma 2.18. We have $\mathbf{L}_{\mathcal{B} G_{s}} \in D_{\text {coh }}^{[0,1]}\left(\mathcal{O}_{\mathcal{B} G_{s}}\right)$

Proof. Consider the map $p: s \rightarrow \mathcal{B} G_{s}$ corresponding to the trivial torsor. The map $p$ is faithfully flat so it suffices to show that $p^{*} \mathbf{L}_{\mathcal{B} G_{s}}$ has cohomology concentrated in degrees 0 and 1 . From the distinguished triangle

$$
p^{*} \mathbf{L}_{\mathcal{B} G_{s}} \rightarrow \mathbf{L}_{s / s} \rightarrow \mathbf{L}_{s / \mathcal{B} G_{s}} \rightarrow p^{*} \mathbf{L}_{\mathcal{B} G_{s}}[1]
$$

and the fact that $\mathbf{L}_{s / s}=0$, we see that $p^{*} \mathbf{L}_{\mathcal{B} G_{s}} \simeq \mathbf{L}_{s / \mathcal{B} G_{s}}[-1]$. Therefore it suffices to show that $\mathbf{L}_{s / \mathcal{B} G_{s}}$ is concentrated in degrees -1 and 0 . Consider the cartesian diagram

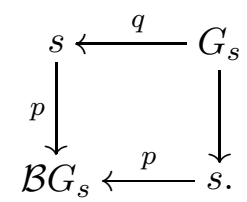

Since $q$ is faithfully flat it then suffices to show that

$$
q^{*} \mathbf{L}_{s / \mathcal{B} G_{s}} \simeq \mathbf{L}_{G_{s} / s}
$$

is concentrated in degrees -1 and 0 . This follows from the fact that $G_{s}$ is a local complete intersection (this can be seen for example from 2.13), and [14, III.3.2.6].

Since $s$ is the spectrum of a field any coherent sheaf on $\mathcal{B} G_{s}$ is locally free, and therefore for any coherent sheaf $\mathcal{F}$ on $\mathcal{B} G_{s}$ we have

$$
\mathcal{R H o m}\left(\mathbf{L}_{\mathcal{B} G_{s}}, \mathcal{F}\right) \in D_{\text {coh }}^{[-1,0]}\left(\mathcal{O}_{\mathcal{B} G_{s}}\right) \text {. }
$$

Since the global section functor is exact on the category $\operatorname{Coh}\left(\mathcal{O}_{\mathcal{B} G_{s}}\right)$ (since $G_{s}$ is linearly reductive) we obtain that

$$
\operatorname{Ext}^{i}\left(\mathbf{L}_{\mathcal{B} G_{s}}, \mathcal{F}\right)=0, \text { for } i \neq-1,0 \text {. }
$$


By [26, 1.5], the obstruction to finding the arrow $\rho_{n+1}$ filling in 2.17.1, is a class in the group

$$
\operatorname{Ext}^{1}\left(\mathbf{L}_{\mathcal{B} G_{s}}, \mathfrak{m}^{n+1} / \mathfrak{m}^{n+2} \otimes_{k(s)} \mathcal{O}_{\mathcal{B} G_{s}}\right)
$$

which by the above is zero. It follows that there exists an arrow $\rho_{n+1}$ filling in 2.17.1.

Method 2: LIFTING USING LiE ALGEBRAS.

Set $G_{n} \stackrel{\text { def }}{=} G_{S_{n}}$ and $G_{n}^{\prime} \stackrel{\text { def }}{=} G_{S_{n}}^{\prime}$.

Let us start from the tautological $G_{0}$-torsor $s=S_{0} \rightarrow \mathcal{B}_{S_{0}} G_{0}$, which we think of as a $G^{\prime}$-torsor via an isomorphism $G_{0} \simeq G_{0}^{\prime}$. Our aim now is to construct a sequence of $G^{\prime}$-torsors $P_{n} \rightarrow \mathcal{B}_{S_{n}} G_{n}$, such that the restriction of each $P_{n}$ to $S_{n-1}$ is isomorphic to the $G^{\prime}$-torsor $P_{n-1} \rightarrow \mathcal{B}_{S_{n-1}} G_{n-1}$.

The Lie algebra $\mathfrak{g}$ of $G_{0}=G_{0}^{\prime}$ is a representation of $G_{0}$, corresponding to a coherent sheaf on $\mathcal{B}_{S_{0}} G_{0}$. It is well known that the obstruction to extending $P_{n-1} \rightarrow \mathcal{B}_{S_{n-1}} G_{n-1}$ to a $G^{\prime}$-torsor lies in the sheaf cohomology $\mathrm{H}^{2}\left(\mathcal{B}_{S_{0}} G_{0},\left(\mathfrak{m}^{n} / \mathfrak{m}^{n+1}\right) \otimes \mathfrak{g}\right) ;$ and this coincides with the cohomology of $G_{0}$ in the representation $\left(\mathfrak{m}^{n} / \mathfrak{m}^{n+1}\right) \otimes \mathfrak{g}$, which is 0 , because $G_{0}$ is linearly reductive.

Each $G^{\prime}$-torsor $P_{n} \rightarrow \mathcal{B}_{S_{n}} G_{n}$ yields a $\left(G, G^{\prime}\right)$-bitorsor

$$
I_{n} \stackrel{\text { def }}{=} S_{n} \times \mathcal{B}_{S_{n}} G_{n} P_{n} \longrightarrow S_{n},
$$

where the morphism $S_{n} \rightarrow \mathcal{B}_{S_{n}} G_{n}$ is the one given by the trivial torsor $G_{n} \rightarrow S_{n}$. So we obtain the desired sequence of bitorsors.

Here is our main result on linearly reductive group schemes.

Theorem 2.19. Let $G \rightarrow S$ be a finite flat group scheme of finite presentation. The following conditions are equivalent.

(a) $G \rightarrow S$ is linearly reductive.

(b) $G \rightarrow S$ is locally well-split.

(c) The fibers of $G \rightarrow S$ are linearly reductive.

(d) The geometric fibers of $G \rightarrow S$ are well-split.

Furthermore, if $S$ is noetherian these conditions are equivalent to either of the following two conditions.

(e) The closed fibers of $G \rightarrow S$ are linearly reductive.

(f) The geometric closed fibers of $G \rightarrow S$ are well-split.

Proof. This follows from Proposition 2.13, Lemma2.11 and Lemma 2.16.

For later use we prove the following.

Lemma 2.20. Let $G \rightarrow \operatorname{Spec} R$ be a linearly reductive group scheme, where $R$ is a henselian local ring. Then there exists a locally diagonalizable normal subgroup $\Delta$ of $G$ such that $G / \Delta$ is étale and tame.

If $R$ is strictly henselian, then $\Delta$ is diagonalizable and $G / \Delta$ is constant. 
Proof. We may assume that $R$ is the henselization of a scheme of finite type over $\mathbb{Z}$ at a point. Set $S=\operatorname{Spec} R$, and let $\mathfrak{m}$ and $k$ be the maximal ideal and the residue field of $R$. Let $\Delta_{0}$ be the connected component of the identity in $G_{k}$; by Lemma 2.11 (国), it is locally diagonalizable. Then we claim that there exists a unique closed subscheme $\Delta \subseteq G$, flat over $S$, whose restriction to $G_{k}$ coincides with $\Delta_{0}$. If $R$ is artinian then $G$ and $G_{k}$ are homeomorphic, and $\Delta$ is simply the connected component of the identity in $G$, which is automatically flat. In general, call $\Delta_{n}$ the connected component of the identity in $G_{S_{n}}$, where $S_{n} \stackrel{\text { def }}{=} \operatorname{Spec} R / \mathfrak{m}^{n+1}$. If $\Delta$ is such a subscheme of $G$, we have $\Delta \cap \Delta_{n}=\Delta_{n}$ for each $n$; hence $\Delta$ is unique. Artin's approximation theorem, applied to the functor that sends each $R$-algebra $A$ into the set of subschemes of $G_{A}$ that are flat over $A$, ensures the existence of such a $\Delta$.

Now we need to show that it is a subgroup scheme, which is equivalent to showing that it is closed under multiplication and inverses. Let $m: G \times G \rightarrow$ $G$ be the multiplication map; $m^{-1}(\Delta) \cap\left(G_{S_{n}} \times G_{S_{n}}\right)$ contains $(\Delta \times \Delta) \cap$ $(G \times G)_{S_{n}}=\Delta_{n} \times \Delta_{n}$ for each $n$, because $\Delta_{n}$ is a subgroup scheme of $G_{S_{n}}$; hence $\Delta \times \Delta \subseteq m^{-1}(\Delta)$ as required. The argument for inverses is similar.

The group scheme of automorphisms of $\Delta_{n}$ over $S_{n}$ is unramified, since its closed fiber is étale. It follows that the image of $\Delta_{n}$ in its automorphism group scheme is trivial, hence $\Delta_{n}$ is abelian.

It is easy to see that each $\Delta_{n}$ is locally diagonalizable, by looking at Cartier duals, and that $G_{n} / \Delta_{n}$ is tame and étale. From this it follows that $\Delta$ is locally diagonalizable and $G / \Delta$ is tame and étale.

The last statement follows from the fact that any étale cover of the spectrum of a strictly henselian ring is trivial.

2.21. A remark on group cohomology. The following is a completely elementary fact, which we explain for lack of a suitable reference.

Let

$$
1 \longrightarrow A \longrightarrow E \longrightarrow G \longrightarrow 1
$$

be an extension of groups, with $A$ abelian. Consider the induced action of $G$ on $A$ by conjugation. Conjugation by an element of $A$ gives an automorphism of $E$, which induces the identity on both $A$ and $G$.

If $\phi: E \rightarrow E$ is another such automorphism, we can consider the function $E \rightarrow A$ defined by $u \mapsto \phi(u) u^{-1}$; this descends to a function $\psi: G \rightarrow A$, linked with $\phi$ by the formula $\phi(u)=\psi([u]) u$, where $[u] \in G$ denotes the image of $u$. It is easy to see that $\psi$ is a crossed homomorphism, and that sending $\phi$ into $\psi$ gives an isomorphism of the group of automorphisms of $E$ that induce the identity on $A$ and on $G$ with the group $\mathrm{Z}^{1}(G, A)$ of crossed homomorphisms. It is also easy to see that $\phi$ is given by conjugation by an element of $A$ if and only if the crossed homomorphism is a boundary. Hence if $\mathrm{H}^{1}(G, A)=0$ the only such automorphisms are obtained by conjugating by elements of $A$. 
2.22. Étale local liftings of linearly reductive group schemes. We will need the following result.

Proposition 2.23. Let $S$ be a scheme, $p \in S$ a point, $G_{0} \rightarrow p$ a linearly reductive group scheme. There exists an étale morphism $U \rightarrow S$, with a point $q \in U$ mapping to $p$, and a linearly reductive group scheme $\Gamma \rightarrow U$ whose restriction $\Gamma_{q} \rightarrow q$ is isomorphic to the pullback of $G_{0}$ to $q$.

Let us start with a lemma. Let $k$ be a field, $G \rightarrow \operatorname{Spec} k$ a well-split group scheme. Let $\Delta$ be the connected component of the identity of $G, H=G / \Delta$. Call $\underline{\mathrm{Aut}}_{k}(G)$ the group scheme representing the functor of automorphisms of $G$ as a group scheme: there is a homomorphism $\Delta \rightarrow \underline{A u t}_{k}(G)$ sending each section of $\Delta$ into the corresponding inner automorphism of $G$; this induces an embedding $\Delta / \Delta^{H} \subseteq \underline{\text { Aut }}_{k}(G)$, where $\Delta^{H}$ denotes the $H$-invariants of $\Delta$.

Lemma 2.24. The connected component of the identity of $\underline{\operatorname{Aut}}_{k}(G)$ is $\Delta / \Delta^{H}$.

Proof. Since $\Delta$ is a characteristic subgroup scheme of $G$, each automorphism of $G_{A} \rightarrow \operatorname{Spec} A$, where $A$ is a $k$-algebra, preserves $\Delta_{A}$. Hence we get homomorphisms of group schemes $\underline{\mathrm{Aut}}_{k}(G) \rightarrow \underline{\mathrm{Aut}}_{k}(\Delta)$ and $\underline{\mathrm{Aut}}_{k}(G) \rightarrow$ $\underline{\text { Aut }}_{k}(H)$, inducing a homomorphism

$$
\underline{\mathrm{Aut}}_{k}(G) \longrightarrow \underline{\mathrm{Aut}}_{k}(\Delta) \times \underline{\mathrm{Aut}}_{k}(H) ;
$$

the kernel of this homomorphism contains $\Delta / \Delta^{H}$. Let us denote by $E$ this kernel; since $\underline{\mathrm{Aut}}_{k}(\Delta) \times \underline{\mathrm{Aut}}_{k}(H)$ is étale over Spec $k$, it is enough to prove that $E$ coincides with $\Delta / \Delta^{H}$.

To do this, we may pass to the algebraic closure of $k$, and assume that $k$ is algebraically closed; then it is enough to prove that given a $k$-algebra $A$, for any element $\alpha \in E(A)$ there exists a faithfully flat extension $A \subseteq A^{\prime}$ such that the image of $\alpha$ in $E\left(A^{\prime}\right)$ comes from $\left(\Delta / \Delta^{H}\right)\left(A^{\prime}\right)$.

By passing to a faithfully flat extension, we may assume that $G(B) \rightarrow$ $H(B)$ is surjective for any $A$-algebra $B$ (because $H$ is constant), so we have an exact sequence

$$
1 \longrightarrow \Delta(B) \longrightarrow G(B) \longrightarrow H(B) \longrightarrow 1 .
$$

Furthermore, again because $H$ is constant, for any $A$-algebra $B$ we have

$$
\Delta^{H}(B)=\Delta(B)^{H(B)} \text {; }
$$

hence for any $B$ we have an injective homomorphism

$$
\Delta(B) / \Delta(B)^{H(B)} \longrightarrow\left(\Delta / \Delta^{H}\right)(B) .
$$

Let us show that $\alpha$ comes from $\left(\Delta / \Delta^{H}\right)(A)$.

Set $B=\Gamma\left(G_{A}, \mathcal{O}\right)$, so that $G_{A}=\operatorname{Spec} B \rightarrow \operatorname{Spec} A$. Then it is easy to see that the natural restriction homomorphism $\operatorname{Aut}_{A}\left(G_{A}\right) \rightarrow \operatorname{Aut}(G(B))$ is injective. The group $\Delta(B)$ has an order that is a power of the characteristic of $k$, while the order of $H(B)$ is prime to the characteristic; so $\mathrm{H}^{1}(H(B), \Delta(B))=0$. By the discussion in 2.21 there exists an element $\delta_{B}$ 
of $\Delta(B)$ whose image in Aut $(G(B))$ (the automorphism given by conjugation by $\delta_{B}$ ) coincides with the image of $\alpha$.

Call $\bar{\delta}_{B}$ the image of $\delta_{B}$ in $\left(\Delta / \Delta^{H}\right)(B)$. We claim that $\bar{\delta}_{B}$ is the image of an element $\bar{\delta}$ of $\left(\Delta / \Delta^{H}\right)(A)$; then the image of $\delta$ in $E(A)$ must be $\alpha$, because $\operatorname{Aut}_{A}\left(G_{A}\right)$ injects into $\operatorname{Aut}(G(B))$.

To prove this, since $\left(\Delta / \Delta^{H}\right)(A)$ is the equalizer of the two natural maps $\left(\Delta / \Delta^{H}\right)(B) \rightrightarrows\left(\Delta / \Delta^{H}\right)\left(B \otimes_{A} B\right)$, it is enough to show that the two images of $\bar{\delta}_{B}$ in $\left(\Delta / \Delta^{H}\right)\left(B \otimes_{A} B\right)$ coincide. The two images of $\delta_{B}$ in $\operatorname{Aut}\left(G\left(B \times_{A} B\right)\right)$ are equal; since $\Delta\left(B \times_{A} B\right) / \Delta\left(B \times_{A} B\right)^{H\left(B \times{ }_{A} B\right)}$ injects into $\operatorname{Aut}\left(G\left(B \times{ }_{A}\right.\right.$ $B)$ ), this implies that the two images of $\delta_{B}$ into $\Delta\left(B \times{ }_{A} B\right)^{H\left(B \times{ }_{A} B\right)}$ coincide. The images of these via the natural injective homomorphism

$$
\Delta\left(B \times{ }_{A} B\right) / \Delta\left(B \times{ }_{A} B\right)^{H\left(B \times{ }_{A} B\right)} \longrightarrow\left(\Delta / \Delta^{H}\right)\left(B \times_{A} B\right)
$$

are the two images of $\bar{\delta}_{B}$, and this completes the proof.

Proof of Proposition 2.23. Let $\overline{k(p)}$ be the algebraic closure of $k(p)$; the pullback $G_{\overline{k(p)}}$ is well-split, that is, it is a semi-direct product $H_{\overline{k(p)}} \ltimes \Delta_{\overline{k(p)}}$, where $H_{\overline{k(p)}}$ is étale, hence a constant group, and $\Delta \overline{k(p)}$ is connected and diagonalizable. This is the pullback of a group scheme $\Gamma=H \ltimes \Delta \rightarrow S$, where $H$ is constant and $\Delta$ is diagonalizable; passing to a Zariski open neighborhood of the image of $p$ in $S$, we may assume that $H$ is tame, so $\Gamma$ is well split. The group scheme $G_{0}$ is a twisted form of the fiber $\Gamma_{p}$. So we need to show that every twisted form of $\Gamma_{p} \rightarrow S$ on a point $p \in S$ extends to an étale neighborhood of $p$. This twisted form is classified by an element of the non-abelian cohomology group $\mathrm{H}_{\mathrm{fppf}}^{1}\left(p, \underline{\mathrm{Aut}}_{k}(\Gamma)\right)$. Let us set $\Delta^{\prime}=\Delta / \Delta^{H}$. The quotient $\underline{\mathrm{Aut}}_{k}(\Gamma) / \Delta_{p}^{\prime}$ is étale, by Lemma 2.24, hence the image of this element in

$$
\mathrm{H}_{\mathrm{fppf}}^{1}\left(p, \underline{\operatorname{Aut}}_{k}(\Gamma) / \Delta_{p}^{\prime}\right)=\mathrm{H}_{\text {ét }}^{1}\left(p, \underline{\mathrm{Aut}}_{k}(\Gamma) / \Delta_{p}^{\prime}\right)
$$

is killed after passing to a finite separable extension of $k(p)$. Any such extension is of the form $k(q)$, where $U \rightarrow S$ is an étale map and $q$ is a point on $U$ mapping on $p$. We can substitute $S$ with $U$, and assume that the image of our element of $\mathrm{H}_{\mathrm{fppf}}\left(p, \underline{\mathrm{Aut}}_{k}(\Gamma)\right)$ in $\mathrm{H}_{\mathrm{fppf}}^{1}\left(p, \underline{\mathrm{Aut}}_{k}(\Gamma) / \Delta_{p}^{\prime}\right)$ is trivial. We have an exact sequence of pointed sets

$$
\mathrm{H}_{\mathrm{fppf}}^{1}\left(p, \Delta_{p}^{\prime}\right) \rightarrow \mathrm{H}_{\mathrm{fppf}}^{1}\left(p, \underline{\operatorname{Aut}}_{k}(\Gamma)\right) \rightarrow \mathrm{H}_{\mathrm{fppf}}^{1}\left(p, \underline{\operatorname{Aut}}_{k}(\Gamma) / \Delta_{p}^{\prime}\right) ;
$$

so our element comes from $\mathrm{H}_{\mathrm{fppf}}^{1}\left(p, \Delta_{p}^{\prime}\right)$. Since $\Delta^{\prime}$ is diagonalizable, it is enough to prove that every element of $\mathrm{H}_{\mathrm{fppf}}^{1}\left(p, \boldsymbol{\mu}_{n}\right)$ comes from $\mathrm{H}_{\mathrm{fppf}}^{1}\left(S, \boldsymbol{\mu}_{n}\right)$, after restricting $S$ in the Zariski topology.

By Kummer theory, every $\boldsymbol{\mu}_{n}$-torsor over $k(p)$ is of the form

$$
\operatorname{Spec} k(p)[t] /\left(t^{n}-a\right) \longrightarrow \operatorname{Spec} k(p)
$$

for some $a \in k(p)^{*}$, with the obvious action of $\boldsymbol{\mu}_{n}$ on $\operatorname{Spec} k(p)[t] /\left(t^{n}-a\right)$. After passing to a Zariski neighborhood of $p \in S$, we may assume that $a$ is the restriction of a section $f \in \mathcal{O}^{*}(S)$. Then the $\boldsymbol{\mu}_{n}$-torsor $\operatorname{Spec}_{S} \mathcal{O}_{S}[t] /\left(t^{n}-\right.$ 
$f) \rightarrow S$ restricts to $\operatorname{Spec} k(p)[t] /\left(t^{n}-a\right) \rightarrow \operatorname{Spec} k(p)$, and this completes the proof.

\section{TAME STACKS}

Let $S$ be a scheme, $\mathcal{M} \rightarrow S$ a locally finitely presented algebraic stack over $S$. We denote by $\mathcal{I} \rightarrow \mathcal{M}$ the inertia group stack; we will always assume that $\mathcal{I} \rightarrow \mathcal{M}$ is finite (and we say that $\mathcal{M}$ has finite inertia). If $T \rightarrow S$ is a morphism, and $\xi$ is an object of $\mathcal{M}(T)$, then the group scheme $\underline{\operatorname{Aut}}_{T}(\xi) \rightarrow T$ is the pullback of $\mathcal{I}$ along the morphism $T \rightarrow \mathcal{M}$ corresponding to $\xi$.

Under this hypothesis, it follows from [16] that there exists a moduli space $\rho: \mathcal{M} \rightarrow M$; the morphism $\rho$ is proper.

Definition 3.1. The stack $\mathcal{M}$ is tame if the functor $\rho_{*}$ : QCoh $\mathcal{M} \rightarrow$ QCoh $M$ is exact.

When $G \rightarrow S$ is a finite flat group scheme, then the moduli space of $\mathcal{B}_{S} G \rightarrow S$ is $S$ itself; so $\mathcal{B}_{S} G$ is tame if and only if $G$ is linearly reductive.

Theorem 3.2. The following conditions are equivalent.

(a) $\mathcal{M}$ is tame.

(b) If $k$ is an algebraically closed field with a morphism Spec $k \rightarrow S$ and $\xi$ is an object of $\mathcal{M}(\operatorname{Spec} k)$, then the automorphism group scheme $\underline{\mathrm{Aut}}_{k}(\xi) \rightarrow$ Spec $k$ is linearly reductive.

(c) There exists an fppf cover $M^{\prime} \rightarrow M$, a linearly reductive group scheme $G \rightarrow M^{\prime}$ acting an a finite and finitely presented scheme $U \rightarrow M^{\prime}$, together with an isomorphism

$$
\mathcal{M} \times_{M} M^{\prime} \simeq[U / G]
$$

of algebraic stacks over $M^{\prime}$.

(d) Same as (ㄷ), but $M^{\prime} \rightarrow M$ is assumed to be étale and surjective.

Corollary 3.3. Let $\mathcal{M}$ be a tame stack over a scheme $S$ and let $\mathcal{M} \rightarrow M$ be its moduli space.

(a) If $M^{\prime} \rightarrow M$ is a morphism of algebraic spaces, then the moduli space of $M^{\prime} \times_{M} \mathcal{M}$ is $M^{\prime}$.

(b) If $\mathcal{M}$ is flat over $S$ then $M$ is also flat over $S$.

Proof. This corollary is proved by standard arguments as follows.

Formation of moduli spaces commutes with flat base change; furthermore, if $\mathcal{M}$ is an algebraic stack locally of finite presentation with finite diagonal, $\mathcal{M} \rightarrow M$ is a morphism into an algebraic space, and $\left\{M_{i} \rightarrow M\right\}$ is an fppf cover, then $M$ is the moduli space of $\mathcal{M}$ if and only if for each $i$ the algebraic space $M_{i}$ is the moduli space of $M_{i} \times_{M} \mathcal{M}$.

Hence by Theorem 3.2 we may assume that $S=\operatorname{Spec} R$ and $M=\operatorname{Spec} A$ are affine, and that $\mathcal{M}$ is of the form $[U / G]$, where $G$ is a linearly reductive group scheme. Then $U$ is finite over $M=U / G$, so it is affine: write $U=$ Spec $B$. Then the group scheme $G$ acts on the $A$-algebra $B$, and $A=B^{G}$. 
Let $N$ be an $R$-module; then there is a natural action of $G$ on $N \otimes_{R} B$, obtained from the action of $G$ on $B$. There is a natural homomorphism $N \otimes_{R} A \rightarrow N \otimes_{R} B$, which is $G$-equivariant, when letting $G$ act trivially on $N \otimes_{R} A$. We claim that the induced homomorphism

$$
N \otimes_{R} A \longrightarrow\left(N \otimes_{R} B\right)^{G}
$$

is an isomorphism. This is obvious when $N$ is free, because in this case $N \otimes_{R} B$ is a direct sum of copies of $B$, with $G$ acting separately on each copy. In general, let $F_{1} \rightarrow F_{0} \rightarrow N \rightarrow 0$ be a free presentation of $N$. We have a commutative diagram

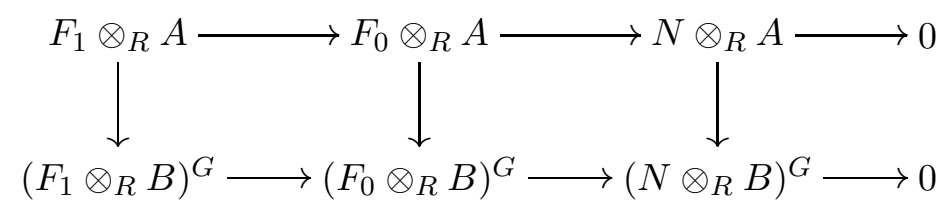

in which both rows are exact (the second one because $G$ is linearly reductive). The first two columns are isomorphisms, hence so is the third.

Part (a) follows easily from this. We need to check that if $M^{\prime} \rightarrow M$ is a morphism, the projection $M^{\prime} \times_{M} \mathcal{M} \rightarrow M^{\prime}$ makes $M^{\prime}$ into the moduli space or $M^{\prime} \times_{M} \mathcal{M}$. We may assume that $M^{\prime}=\operatorname{Spec} A^{\prime}$ is affine, and that $S=M$, so that $R=M$; then the statement follows from the fact the natural homomorphism $A^{\prime}=A^{\prime} \otimes_{A} A \rightarrow\left(A^{\prime} \otimes_{A} B\right)^{G}$ is an isomorphism.

Part (b) is also easy. Assume that $B$ is flat over $R$. The isomorphism $N \otimes_{R} A \simeq\left(N \otimes_{R} B\right)^{G}$ is functorial in the $R$-module $N$, and both tensoring with $B$ and taking invariants give exact functors.

Corollary 3.4. If $\mathcal{M} \rightarrow S$ tame and $S^{\prime} \rightarrow S$ is a morphism of schemes, then $S^{\prime} \times_{S} \mathcal{M}$ is a tame stack over $S^{\prime}$.

Corollary 3.5. The stack $\mathcal{M} \rightarrow S$ tame if and only if for any morphism Spec $k \rightarrow S$, where $k$ is an algebraically closed field, the geometric fiber Spec $k \times{ }_{S} \mathcal{M}$ is tame.

Proof of Theorem 3.2. It is obvious that (d) implies (IC). It is straightforward to see that (드) implies both (国) and (b).

Let us check that (a) implies (b). Let Spec $k \rightarrow \mathcal{M}$ be the morphism corresponding to the object $\xi$ of $\mathcal{M}(\operatorname{Spec} k)$; set $G=\underline{\operatorname{Aut}}_{k}(\xi)$. Call $\mathcal{M}_{0}$ the pullback Spec $k \times{ }_{M} \mathcal{M}$; this admits a section Spec $k \rightarrow \mathcal{M}_{0}$, and the residual gerbe of this section, which is a closed substack of $\mathcal{M}_{0}$, is isomorphic to $\mathcal{B}_{k} G$. So we get a commutative (non cartesian) diagram

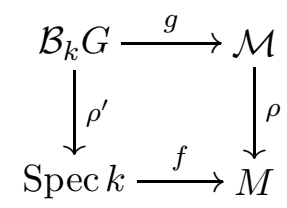


whose rows are affine. Hence we have that $g_{*}: \mathrm{QCoh}\left(\mathcal{B}_{k} G\right) \rightarrow \mathrm{Q} \operatorname{Coh}(\mathcal{M})$ is an exact functor, while $\rho_{*}: \mathrm{QCoh}(\mathcal{M}) \rightarrow \mathrm{QCoh}(M)$ is exact by definition. Also we have an equality of functors $f_{*} \rho_{*}^{\prime}=\rho_{*} g_{*}$; hence, if

$$
0 \longrightarrow V_{1} \longrightarrow V_{2} \longrightarrow V_{3} \longrightarrow 0
$$

is an exact sequence of representations of $G$, considered as an exact sequence of quasi-coherent sheaves on $\mathcal{B}_{k} G$, we have that the sequence

$$
0 \longrightarrow f_{*}\left(V_{1}^{G}\right) \longrightarrow f_{*}\left(V_{2}^{G}\right) \longrightarrow f_{*}\left(V_{3}^{G}\right) \longrightarrow 0
$$

is exact; and this implies that

$$
0 \longrightarrow V_{1}^{G} \longrightarrow V_{2}^{G} \longrightarrow V_{3}^{G} \longrightarrow 0
$$

is exact. Hence $G$ is linearly reductive, as claimed.

Now let us prove that (b) implies (d) . In fact, we will prove a stronger version of this implication.

Proposition 3.6. Let $\mathcal{M} \rightarrow S$ be a locally finitely presented algebraic stack with finite inertia and moduli space $\rho: \mathcal{M} \rightarrow M$. Let $k$ be a field with a morphism Spec $k \rightarrow S$, and let $\xi$ be an object of $\mathcal{M}(\operatorname{Spec} k)$; assume that the automorphism group scheme $\underline{\mathrm{Aut}}_{k}(\xi) \rightarrow$ Spec $k$ is linearly reductive. Denote by $p \in M$ the image of the composite Spec $k \rightarrow \mathcal{M} \rightarrow M$. Then there exists an étale morphism $U \rightarrow M$ having $p$ in its image, a linearly reductive group scheme $G \rightarrow U$ acting on a finite scheme $V \rightarrow U$ of finite presentation, and an isomorphism $[V / G] \simeq U \times_{S} \mathcal{M}$ of algebraic stacks over $U$.

Thus, if $\mathcal{M}$ has an object over a field with linearly reductive automorphism group, then there is an open tame substack of $\mathcal{M}$ (the image of $U$ ) containing this object.

Proof. The proof is divided into three steps.

We may assume that $M$ is affine and of finite type over $\mathbb{Z}$.

THE CASE $k=k(p)$. We start by assuming that the residue field $k(p)$ of $p \in M$ equals $k$. After passing to an étale cover of $M$, we may also assume that $\underline{\mathrm{Aut}}_{k}(\xi)$ extends to a linearly reductive group scheme $G \rightarrow M$ (Proposition 2.23).

By standard limit arguments we may assume that $M$ is the spectrum of a local henselian ring $R$ with residue field $k$. The result follows once we have shown that there is a representable morphism $\mathcal{M} \rightarrow \mathcal{B}_{M} G$ of algebraic stacks, equivalently, a $G$-torsor $P \rightarrow \mathcal{M}$ in which the total space is an algebraic space.

Let us denote by $\mathcal{M}_{0}$ the residual gerbe $\mathcal{B}_{k} \underline{\operatorname{Aut}}_{k}(\xi)=\mathcal{B}_{k} G_{p}$; this is a closed substack of $\mathcal{M}$, having Spec $k$ as its moduli space. This closed substack gives a sheaf of ideals $\mathcal{I} \subseteq \mathcal{O}_{\mathcal{M}}$; we denote by $\mathcal{M}_{n}$ the closed substack of $\mathcal{M}$ whose sheaf of ideals is $\mathcal{I}^{n+1}$. Denote by $\mathfrak{g}$ the Lie algebra of $\underline{\mathrm{Aut}}_{k}(\xi)$. 
The obstruction to extending a $G$-torsor $P_{n-1} \rightarrow \mathcal{M}_{n-1}$ to a $G$-torsor $P_{n} \rightarrow \mathcal{M}_{n}$ lies in

$$
\begin{aligned}
\mathrm{H}^{2}\left(\mathcal{M}_{0},\left(\mathcal{I}^{n} / \mathcal{I}^{n+1}\right) \otimes \mathfrak{g}\right) & =\mathrm{H}^{2}\left(G_{p},\left(\mathcal{I}^{n} / \mathcal{I}^{n+1}\right) \otimes \mathfrak{g}\right) \\
& =0 ;
\end{aligned}
$$

Alternatively, in terms of the cotangent complex, the obstruction lies in $\operatorname{Ext}^{1}\left(\mathbf{L} g^{*} \mathbf{L}_{\mathcal{B}_{k} G_{p}}, \mathcal{I}^{n} / \mathcal{I}^{n+1}\right)=0$, where $g: \mathcal{M}_{0} \rightarrow \mathcal{B}_{M} G$ is the morphism defined by $P_{0}$.

Hence we can construct a sequence of $G$-torsors $P_{n} \rightarrow \mathcal{M}_{n}$, such that the restriction of $P_{n}$ to $P_{n-1}$ is isomorphic to $P_{n-1}$, and such that the torsor $P_{0} \rightarrow \mathcal{M}_{0}$ has Spec $k$ as its total space.

Let $\mathfrak{m}$ be the maximal ideal of $R$, and set $M_{n}=\operatorname{Spec} R / \mathfrak{m}^{n+1}$. The systems of ideals $\left\{\mathcal{I}^{n}\right\}$ and $\mathfrak{m}^{n} \mathcal{O}_{\mathcal{M}}$ are cofinal; hence we get a sequence of $G$-torsors $Q_{n} \rightarrow M_{n} \times M \mathcal{M}$, such that the restriction of $Q_{n}$ to $Q_{n-1}$ is isomorphic to $Q_{n-1}$, and such that the restriction of $Q_{0}$ to $\mathcal{M}_{0}$ has Spec $k$ as its total space. We can define a functor from $R$-algebras to sets that sends each $R$-algebra $A$ to the set of isomorphism classes of $G$-torsors on the stack $\mathcal{M}_{A}$. This functor is easily checked to be limit-preserving (for example, by using a presentation of $\mathcal{M}$ and descent for $G$-torsors). So we can apply Artin's approximation theorem, and conclude that there exists a $G$-torsor on $\mathcal{M}$, whose restriction to $\mathcal{M}_{0}$ has Spec $k$ as its total space.

The total space $\mathcal{P}$ is an algebraic stack with finite inertia; furthermore, the inverse image of $\mathcal{M}_{0}$ in $\mathcal{P}$ is isomorphic to Spec $k$. The locus where the inertia stack $\mathcal{I}_{\mathcal{P}} \rightarrow \mathcal{P}$ has fiber of length larger than 1 is a closed substack of $\mathcal{P}$, whose image in $M=\operatorname{Spec} R$ is a closed subscheme that does not contain $p$; hence this locus is empty. So $\mathcal{P}$ is an algebraic space (in fact an affine scheme); and this concludes the proof of the first case.

ObTAining a flat MORPhism AND PROOF of PART (IC). Now we prove a weaker version of the Proposition, with the same statement, except that the morphism $U \rightarrow M$ is only supposed to be flat and finitely presented, instead of étale. This is sufficient to prove that part (C) holds.

By passing to the algebraic closure of $k$ we may assume that $k$ is algebraically closed.

We claim that there exists a finite extension $k^{\prime}$ of the residue field $k(p)$ contained in $k$, such that the object $\xi$ is defined over $k^{\prime}$. In fact, it follows from the definition of moduli space that there exists an object $\eta$ of $\operatorname{Spec} \overline{k(p)}$ whose pullback to Spec $k$ is isomorphic to $\xi$. This $\eta$ gives an object of the algebraic stack Spec $k(p) \times_{M} \mathcal{M}$ over Spec $\overline{k(p)}$, which is finitely presented over Spec $k(p)$, and any such object is defined over a finite extension $k^{\prime}$ of $k(p)$. Hence we may assume that $k$ is a finite extension of $k(p)$.

There is a flat morphism of finite presentation $M^{\prime} \rightarrow M$, with a point $q \in U$ mapping to $p$, such that $k(q)=k$ [10, $\left.0_{I I I} .10 .3\right]$; hence, by applying the first step to $M^{\prime} \times_{M} \mathcal{M}$, there is an étale morphism $U \rightarrow M^{\prime}$ containing $q$ in its image, such that $U \times_{M} \mathcal{M}$ has the required quotient form. 
The CONCLusion. The argument of the proof of the previous case shows that to conclude we only need the following fact.

Proposition 3.7. Let $\mathcal{M} \rightarrow S$ be a tame stack with moduli space $\rho: \mathcal{M} \rightarrow$ $M, k$ a field. Given a morphism Spec $k \rightarrow M$, there exists a finite separable extension $k \subseteq k^{\prime}$ and a lifting Spec $k^{\prime} \rightarrow \mathcal{M}$ of the composite Spec $k^{\prime} \rightarrow$ Spec $k \rightarrow M$.

Proof. We are going to need some preliminaries.

Suppose that $\mathcal{X}$ and $\mathcal{Y}$ are algebraic stacks over a scheme $S$; consider the stack $\underline{\operatorname{Hom}}_{S}(\mathcal{X}, \mathcal{Y})$ whose sections over an $S$-scheme $T$ are morphisms of $T$-stacks $\mathcal{X}_{T} \rightarrow \mathcal{Y}_{T}$ (see [24]). We will denote by $\underline{\operatorname{Hom}}_{S}^{\mathrm{rep}}(\mathcal{X}, \mathcal{Y})$ the substack whose sections are representable morphisms $\mathcal{X}_{T} \rightarrow \mathcal{Y}_{T}$.

Lemma 3.8. Let $G$ and $H$ be finite and finitely presented flat group schemes over a locally noetherian scheme $S$. Then the stacks $\underline{\operatorname{Hom}}_{S}\left(\mathcal{B}_{S} G, \mathcal{B}_{S} H\right)$ and $\underline{\operatorname{Hom}}_{S}^{\mathrm{rep}}\left(\mathcal{B}_{S} G, \mathcal{B}_{S} H\right)$ are finitely presented over $S$.

Proof. By standard arguments, we may assume that $S$ is affine and finitely generated over $\mathbb{Z}$.

Consider the fppf sheaf $\underline{\operatorname{Hom}}_{S}(G, H)$ whose sections over an $S$-scheme $T$ are homomorphism of group schemes $G_{T} \rightarrow H_{T}$. This is a locally closed subscheme of the Hilbert scheme of $G \times{ }_{S} H$ over $S$, hence it is of finite type. Furthermore, consider the subsheaf $\operatorname{Hom}_{S}^{\text {inj }}(G, H)$ consisting of universally injective homomorphisms $G_{T} \rightarrow H_{T}$ (a homomorphism is universally injective if it is injective as a homomorphism of sheaves in the fppf topology, and it stays such after arbitrary base change on $T$ ). We claim that the embedding $\underline{\operatorname{Hom}}_{S}^{\text {inj }}(G, H) \subseteq \underline{\operatorname{Hom}}_{S}(G, H)$ is represented by an open subscheme of $\underline{\operatorname{Hom}}_{S}(G, H)$.

To see this, consider the universal object $\phi: G_{T} \rightarrow H_{T}$, where we have set $T \stackrel{\text { def }}{=} \underline{\operatorname{Hom}}_{S}(G, H)$, and its kernel $K \subseteq G_{T}$. The sheaf $K$ is a (not necessarily flat) finite group scheme on $T$. Formation of $K$ commutes with base change on $T$; hence the fiber product $\underline{\operatorname{Hom}}_{S}^{\text {inj }}(G, H)$ is represented by the open subscheme of $T$ consisting of point $t \in T$ such that $K_{t}$ has length 1 .

There is a natural action by conjugation of $H$ on $\underline{\operatorname{Hom}}_{S}(G, H)$, preserving $\underline{\operatorname{Hom}}_{S}^{\text {inj }}(G, H)$. We claim that $\underline{\operatorname{Hom}}_{S}\left(\mathcal{B}_{S} G, \mathcal{B}_{S} H\right)$ is isomorphic to the quotient stack $\left[\underline{\operatorname{Hom}}_{S}(G, H) / H\right]$, and that $\underline{\operatorname{Hom}}_{S}^{\mathrm{rep}}\left(\mathcal{B}_{S} G, \mathcal{B}_{S} H\right)$ is the open substack $\left[\underline{\operatorname{Hom}}_{S}^{\mathrm{inj}}(G, H) / H\right]$. This is obviously enough to prove the statement.

Let us start by producing a functor

$$
\Phi: \underline{\operatorname{Hom}}_{S}\left(\mathcal{B}_{S} G, \mathcal{B}_{S} H\right) \longrightarrow\left[\underline{\operatorname{Hom}}_{S}(G, H) / H\right] .
$$

For each $S$-scheme $T$ and each base-preserving functor $F: \mathcal{B}_{T} G_{T} \rightarrow \mathcal{B}_{T} H_{T}$ we need to exhibit an $H$-torsor $Q \rightarrow T$ and an $H$-equivariant morphism $Q \rightarrow \underline{\operatorname{Hom}}_{S}(G, H)$. The torsor $Q \rightarrow T$ is simply the image via $F$ of the trivial $G$-torsor $G_{T} \rightarrow T$. Furthermore, the functor $F$ induces a homomorphism from $\underline{A u t}_{S}\left(G_{Q} \rightarrow Q\right)=G_{Q}$ to the automorphism group scheme of the 
image of $G_{Q} \rightarrow Q$ in $\mathcal{B}_{Q} H_{Q}$. Since this image is the pullback of $Q$ to $Q$, which is canonically a trivial torsor, its automorphism group scheme is $H_{Q}$. This defines an object of $\underline{\operatorname{Hom}}_{S}(G, H)(Q)$, hence a morphism $Q \rightarrow$ $\underline{\operatorname{Hom}}_{S}(G, H)$; this is easily seen to be $H$-equivariant. This gives an object of $\left[\underline{\operatorname{Hom}}_{S}(G, H) / H\right](Q)$ : the functor $\Phi$ will send $F$ to this object. We leave it to the reader to complete the definition of $\Phi$ by defining its action on arrows.

The functor

$$
\Psi:\left[\underline{\operatorname{Hom}}_{S}(G, H) / H\right] \longrightarrow \underline{\operatorname{Hom}}_{S}\left(\mathcal{B}_{S} G, \mathcal{B}_{S} H\right)
$$

is defined as follows. Take an object of $\left.\underline{\operatorname{Hom}}_{S}(G, H) / H\right](T)$, that is, a principal $H$-bundle $Q \rightarrow T$, with an $H$-equivariant morphism $\phi: Q \rightarrow$ $\underline{\operatorname{Hom}}_{S}(G, H)$. We need to define a base-preserving functor $\mathcal{B}_{T} G_{T} \rightarrow \mathcal{B}_{T} H_{T}$. Suppose that $T^{\prime}$ is a $T$-scheme and $P \rightarrow T^{\prime}$ is a $G$-torsor. We can define an action of $G$ on $P \times_{T} Q$ by the formula

$$
(p, q) g=(p g, q \phi(q)(g)) .
$$

Using the formula

$$
\phi(q h)=h^{-1} \phi(q) h
$$

which expresses the fact that $\phi$ is $H$-equivariant, we check that this action of $G$ commutes with the obvious action of $H$ defined by $(p, q) h=(p, q h)$. The resulting action of $G \times H$ is free and $\left(P \times_{T} Q\right) /\left(G \times{ }_{S} H\right)=T^{\prime}$, so $\left(P \times_{T} Q\right) / G \rightarrow P / G=T^{\prime}$ is an $H$-torsor.

We set $\Psi(Q, \phi)\left(P \rightarrow T^{\prime}\right)=\left(P \times_{T} Q\right) / G \rightarrow T^{\prime}$. This defines the action of $\Psi$ on objects; the definition of the action of $\Psi$ on arrows is straightforward.

Let us verify that $\Phi \Psi$ is isomorphic to $\operatorname{id}_{\left[\underline{\operatorname{Hom}}_{S}(G, H) / H\right]}$. Let $T$ be an $S$ scheme, $Q \rightarrow T$ an $H$-torsor, $\phi: Q \rightarrow \underline{\operatorname{Hom}}_{S}(G, H)$ an $H$-equivariant maps. Set $\Phi \Psi(Q, \phi)=\left(Q^{\prime}, \phi^{\prime}\right)$; we need to produce an isomorphism between $(Q, \phi)$ and $\left(Q^{\prime}, \phi^{\prime}\right)$.

By definition, $Q^{\prime}$ is the quotient $\left(G \times{ }_{S} Q\right) / G$, where the action of $G$ on $G \times{ }_{S} Q$ is defined by the formula $(g, q) g_{1}=\left(g g_{1}, q \phi(q)\left(g_{1}\right)\right)$. It is immediate to verify that the morphism $G \times{ }_{S} Q \rightarrow Q$ is $G$-invariant and $H$-equivariant; hence the induced morphism $Q^{\prime}=\left(G \times{ }_{S} Q\right) / G \rightarrow Q$ is an isomorphism of $H$-torsors. We leave it to the reader to check that the homomorphism $\phi^{\prime}: Q^{\prime} \rightarrow \underline{\operatorname{Hom}}_{S}(G, H)$ induced by $\Psi(Q, \phi)$ corresponds to $\phi$; this gives the natural isomorphism $(Q, \phi) \simeq\left(Q^{\prime}, \phi^{\prime}\right)$.

Next we need to check that $\Psi \Phi$ is isomorphic to $\operatorname{id}_{\underline{\operatorname{Hom}}_{S}\left(\mathcal{B}_{S} G, \mathcal{B}_{S} H\right)}$. Let $F: \mathcal{B}_{T} G \rightarrow \mathcal{B}_{T} H$ be a base-preserving functor. Set $\overline{\Phi F}=(Q \rightarrow T, \phi)$; by definition, $Q \rightarrow T=F\left(G_{T} \rightarrow T\right)$. We need to produce a canonical isomorphism between $F$ and $\Psi(Q \rightarrow T, \phi)$. Let $T^{\prime}$ be a $T$-scheme, $P \rightarrow T^{\prime}$ a $G$-torsor; we need an isomorphism of $H$-torsors between $Q^{\prime} \rightarrow T \stackrel{\text { def }}{=} F(P \rightarrow$ $\left.T^{\prime}\right)$ and $\Psi(Q \rightarrow T)\left(P \rightarrow T^{\prime}\right)$. By definition, $\Psi(Q \rightarrow T)\left(P \rightarrow T^{\prime}\right)$ is the $H$-torsor $\left(P \times_{T} Q\right) / G \rightarrow T^{\prime}$. There is a morphism $P \times_{T} Q \rightarrow Q^{\prime}$ which is defined as follows. If $T^{\prime \prime}$ is a $T^{\prime}$-scheme, a morphism $T^{\prime \prime} \rightarrow P$ corresponds 
to a morphism $G_{T^{\prime \prime}} \rightarrow P$ of $G$-torsors over $T^{\prime \prime}$. This in turn induces a morphism of $H$-torsors

$$
T^{\prime \prime} \times_{T} Q=F\left(G_{T^{\prime \prime}} \rightarrow T^{\prime \prime}\right) \longrightarrow T^{\prime \prime} \times_{T^{\prime}} Q^{\prime} .
$$

This construction defines a morphism $P \times_{T} Q \rightarrow Q^{\prime}$. This is easily checked to be $G$-invariant and $H$-equivariant: hence it defines the desired isomorphism of $H$-torsors $\left(P \times_{T} Q\right) / G \simeq Q^{\prime}$.

We have left to prove that $\underline{\operatorname{Hom}}_{S}^{\mathrm{rep}}\left(\mathcal{B}_{S} G, \mathcal{B}_{S} H\right)$ is isomorphic to the quotient $\left[\underline{\operatorname{Hom}}_{S}^{\mathrm{inj}}(G, H) / H\right]$, or, equivalently, that the inverse image of the substack $\underline{\operatorname{Hom}}_{S}^{\mathrm{rep}}\left(\mathcal{B}_{S} G, \mathcal{B}_{S} H\right) \subseteq \underline{\operatorname{Hom}}_{S}\left(\mathcal{B}_{S} G, \mathcal{B}_{S} H\right)$ into $\underline{\operatorname{Hom}}_{S}(G, H)$ equals $\underline{\operatorname{Hom}}_{S}^{\text {inj }}(G, H)$. But this follows immediately from the well known fact that a morphism $F: \mathcal{B}_{T} G_{T} \rightarrow \mathcal{B}_{T} H_{T}$ is representable if and only if for any $T^{\prime} \rightarrow T$ and any object $\xi$ of $\mathcal{B}_{S} G\left(T^{\prime}\right)$, the induced homomorphism from Aut $_{T^{\prime}}(\xi) \rightarrow$ Aut $_{T^{\prime}}(F(\xi))$ is injective.

Let $k$ be a field, $R$ be an artinian local $k$-algebra with residue field $k$, $G$ a linearly reductive group scheme acting on $R$. Set $\mathcal{M}=[\operatorname{Spec} R / G]$, and assume that the moduli space of $\mathcal{M}$ is $\operatorname{Spec} k$ (this is equivalent to assuming that $\left.R^{G}=k\right)$. We have a natural embedding $\mathcal{B}_{k} G=[\operatorname{Spec} k / G] \subseteq$ $[\operatorname{Spec} R / G]=\mathcal{M}$.

Lemma 3.9. If $T$ is a $k$-scheme, any representable morphism of $k$-stacks $\rho: \mathcal{B}_{k} G \times_{\text {Spec } k} T \rightarrow \mathcal{M}$ factors through $\mathcal{B}_{k} G \subseteq \mathcal{M}$.

Proof. Let $P$ be the pullback of $\operatorname{Spec} R \rightarrow \mathcal{M}$ to $\mathcal{B}_{k} G \times \times_{\text {Spec } k} T$; then $P$ is an algebraic space (since $\rho$ is representable) with an action of $G$, such that the morphism $P \rightarrow \operatorname{Spec} R$ is $G$-equivariant. We claim that the composite $P \rightarrow \mathcal{B}_{k} G \times T \rightarrow T$ is an isomorphism.

Since it is finite and flat it enough to prove that is an isomorphism when pulled back to a geometric point $\operatorname{Spec} \Omega \rightarrow T$, were $\Omega$ is an algebraically closed field; so we may assume that $T=\operatorname{Spec} \Omega$. Choose a section $\operatorname{Spec} \Omega \rightarrow$ $P:$ since there is a unique morphism $\operatorname{Spec} \Omega \rightarrow \mathcal{B}_{\Omega} G_{\Omega}$ over $\Omega$, we get a commutative diagram

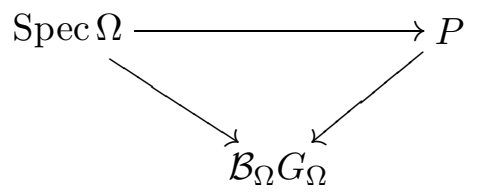

Since both Spec $\Omega \rightarrow \mathcal{B}_{\Omega} G_{\Omega}$ and $P \rightarrow \mathcal{B}_{\Omega} G_{\Omega}$ are $G$-torsors, the degrees of both over $\mathcal{B}_{\Omega} G_{\Omega}$ equal the order of $G$; hence $\operatorname{Spec} \Omega \rightarrow P$ is an isomorphism, and $P \rightarrow$ Spec $\Omega$ is its inverse.

Thus, since the composite $P \rightarrow \mathcal{B}_{k} G \times_{\text {Spec } k} T \rightarrow T$ is $G$-equivariant, this means that the action of $G$ on $P$ is trivial. The morphism $P \rightarrow \operatorname{Spec} R$ corresponds to a ring homomorphism $R \rightarrow \mathcal{O}(P)$, which is $G$-equivariant, and the action of $G$ on $\mathcal{O}(P)$ is trivial. But if $\mathfrak{m}$ is the maximal ideal of $R$, there is a splitting of $G$-modules $R \simeq \mathfrak{m} \oplus k$; and $\mathfrak{m}^{G}=0$, because 
$R^{G}=k$. So $\mathfrak{m}$ is a sum of non-trivial irreducible representations, since $G$ is linearly reductive, and any $G$-equivariant linear map $\mathfrak{m} \rightarrow \mathcal{O}(P)$ is trivial. So $P \rightarrow \operatorname{Spec} R$ factors through Spec $k$, so $\rho: \mathcal{B}_{k} G \times \times_{\text {Spec } k} T \rightarrow \mathcal{M}$ factors through $[\operatorname{Spec} k / G]=\mathcal{B}_{k} G$, as claimed.

Let us prove Proposition 3.7. Since $\mathcal{M}$ is limit-preserving, it is sufficient to show that any morphism Spec $k \rightarrow M$, where $k$ is a separably closed field, lifts to Spec $k \rightarrow \mathcal{M}$.

Notice that Corollary 3.3 (b) can now be applied, since its proof only requires part (ㄷ) of the theorem, which we have just verified. Hence the moduli space of Spec $k \times_{M} \mathcal{M}$ is $\operatorname{Spec} k$, and we can base change to Spec $k$, and assume that $M=\operatorname{Spec} k$.

Let $k \subseteq k^{\prime}$ be a finite field extension such that $\mathcal{M}\left(k^{\prime}\right)$ is non-empty. Pick an object $\xi \in \mathcal{M}\left(k^{\prime}\right)$, and set $G_{k^{\prime}}=\underline{\operatorname{Aut}}_{k^{\prime}}(\xi)$. After extending $k^{\prime}$, we may assume that $G_{k^{\prime}}$ is of the form $H_{k^{\prime}} \ltimes \Delta_{k^{\prime}}$, where $\Delta_{k^{\prime}}$ is a diagonalizable group scheme whose order is a power of the characteristic of $k$ and $H_{k^{\prime}}$ is a constant tame group scheme. There exist unique group schemes $\Delta$ and $H$, respectively diagonalizable and constant, whose pullbacks to Spec $k^{\prime}$ coincide with $\Delta_{k^{\prime}}$ and $H_{k^{\prime}}$; furthermore, the action of $H_{k^{\prime}}$ on $\Delta_{k^{\prime}}$ comes from a unique action of $H$ on $\Delta$. We set $G=H \ltimes \Delta$ : this $G$ is a group scheme on Spec $k$ inducing $G_{k^{\prime}}$ by base change.

Lemma 3.10. The stack $\underline{\operatorname{Hom}}_{k}^{\mathrm{rep}}\left(\mathcal{B}_{k} G, \mathcal{M}\right)$ is of finite type over $k$.

Proof. It is enough to prove the result after base changing to $k^{\prime}$; we can therefore assume that $\mathcal{M}=[\operatorname{Spec} R / G]$, where $R$ is an artinian $k$-algebra with residue field $k$, because of the first part of the proof. Then by Lemma 3.9 the stack $\underline{\operatorname{Hom}}_{k}^{\mathrm{rep}}\left(\mathcal{B}_{k} G, \mathcal{M}\right)$ is isomorphic to

$$
\underline{\operatorname{Hom}}_{k}^{\mathrm{rep}}\left(\mathcal{B}_{k} G, \mathcal{B}_{k} G\right)
$$

which is of finite type by Lemma 3.8 .

The morphism $t:$ Spec $k \rightarrow \mathcal{B}_{k} G$ corresponding to the trivial torsor induces a morphism

$$
F: \underline{\operatorname{Hom}}_{k}^{\mathrm{rep}}\left(\mathcal{B}_{k} G, \mathcal{M}\right) \longrightarrow \mathcal{M}
$$

by composition with $t$.

Consider the scheme-theoretic image $\underline{\mathcal{M}} \subseteq \mathcal{M}$ of the morphism $F$ : this is the smallest closed substack of $\mathcal{M}$ with the property that $F^{-1}(\underline{\mathcal{M}})=$ $\underline{\operatorname{Hom}}_{k}^{\text {rep }}\left(\mathcal{B}_{k} G, \mathcal{M}\right)$. Its sheaf of ideals is the kernel of the homomorphism $\mathcal{O}_{\mathcal{M}} \rightarrow F_{*} \mathcal{O}_{\underline{\mathrm{Hom}}_{k}^{\mathrm{rep}}\left(\mathcal{B}_{k} G, \mathcal{M}\right)}$.

Lemma 3.11. We have

$$
\operatorname{Spec} k^{\prime} \times_{\operatorname{Spec}} k \underline{\mathcal{M}}=\mathcal{B}_{k} G_{k^{\prime}} \subseteq \mathcal{M}_{k^{\prime}},
$$

where $\mathcal{B}_{k^{\prime}} G_{k^{\prime}}$ is embedded in $\mathcal{M}_{k^{\prime}}$ as the residual gerbe of $\xi$. 
Proof. By the first part of the proof, we can write $\mathcal{M}_{k^{\prime}}$ in the form $\left[\operatorname{Spec} R / G_{k^{\prime}}\right]$, where $R$ is an artinian $k$-algebra with residue field $k^{\prime}$. Formation of schemetheoretic images commutes with flat base change, hence we need to show that the scheme-theoretic image of the morphism

$$
F_{k^{\prime}}: \underline{\operatorname{Hom}}_{k^{\prime}}^{\mathrm{rep}}\left(\mathcal{B}_{k^{\prime}} G_{k^{\prime}}, \mathcal{M}_{k^{\prime}}\right) \longrightarrow \mathcal{M}_{k^{\prime}}
$$

is equal to $\mathcal{B}_{k^{\prime}} G_{k^{\prime}}$; or, equivalently, that for any morphism

$$
g: T \longrightarrow \underline{\operatorname{Hom}}_{k^{\prime}}^{\mathrm{rep}}\left(\mathcal{B}_{k^{\prime}} G_{k^{\prime}}, \mathcal{M}_{k^{\prime}}\right)
$$

the composite $F_{k^{\prime}} \circ g: T \rightarrow \mathcal{M}_{k^{\prime}}$ factors through $\left[\operatorname{Spec} k^{\prime} / G_{k^{\prime}}\right]$. This follows from Lemma 3.9 .

Now we can replace $\mathcal{M}$ with $\underline{\mathcal{M}}$, and assume that $\mathcal{M}_{k^{\prime}}$ is $\mathcal{B}_{k^{\prime}} G_{k^{\prime}}$. It follows that $\mathcal{M}$ is a gerbe in the fppf topology over Spec $k$.

Next we define an étale gerbe $\mathcal{N}$, with a morphism $\mathcal{G} \rightarrow \mathcal{N}$.

For any $k$-scheme $T$ and any object $\xi \in \mathcal{M}(T)$, the automorphism group scheme $G_{\xi} \rightarrow T$ is linearly reductive; let

$$
1 \longrightarrow \Delta_{\xi} \longrightarrow G_{\xi} \longrightarrow H_{\xi} \longrightarrow 1
$$

be the connected étale sequence of $G_{\xi}$. More concretely, $\Delta_{\xi}$ is the subfunctor of $G_{\xi}$ of automorphisms whose order is a power of the characteristic of $k$. If $f: T_{1} \rightarrow T$ is a morphism of schemes, then $G_{f^{*} \xi}=T_{1} \times_{T} G_{\xi}$ (this is a general property of fibered categories), and $\Delta_{f^{*} \xi}=T_{1} \times_{T} \Delta_{\xi}$. We define $\mathcal{N}$ to be the stack $\mathcal{M} \rrbracket \Delta$, whose existence is assured by Theorem A.1. We claim that $\mathcal{N}$ is a Deligne-Mumford stack. It is enough to check that for any algebraically closed field $k$ and any object $\bar{\xi}$ of $\mathcal{N}($ Spec $k)$, the automorphism group scheme $\underline{\mathrm{Aut}}_{k}(\bar{\xi})$ is reduced. However, since the morphism $\mathcal{M} \rightarrow \mathcal{N}$ is of finite type, the object $\bar{\xi}$ comes from an object $\xi$ of $\mathcal{M}(\operatorname{Spec} k)$, and we have

$$
\underline{\operatorname{Aut}}_{k}(\bar{\xi})=G_{\xi} / \Delta_{\xi}
$$

this is reduced by definition.

The pullback of $\mathcal{N}$ to Spec $k^{\prime}$ is $\mathcal{B}_{k^{\prime}}\left(G_{k^{\prime}} / \Delta_{k^{\prime}}\right)$ (recall that $\Delta_{k^{\prime}}$ is the connected component of the identity in $\left.G_{k^{\prime}}\right)$; so $\mathcal{N}$ is an étale gerbe over Spec $k$. Since $k$ is separably closed, there is a $k$-morphism $\operatorname{Spec} k \rightarrow \mathcal{N}$. We can replace $\mathcal{M}$ by $\mathcal{M} \times_{\mathcal{N}}$ Spec $k$, so that $\mathcal{M}_{k^{\prime}}=\mathcal{B}_{k^{\prime}} \Delta_{k^{\prime}}$.

In this case, we claim that $\mathcal{M}$ is banded by the diagonalizable group $\Delta \rightarrow$ Spec $k$ (recall that we have defined this as the diagonalizable group scheme whose pullback to Spec $k^{\prime}$ is $\Delta_{k^{\prime}}$ ). In fact, since $\mathcal{M}$ is a gerbe, and all of its objects have abelian automorphism groups, then the automorphism group schemes descend to a group scheme over Spec $k$, whose pullback to Spec $k^{\prime}$ is $\Delta_{k^{\prime}}$. So this group scheme is a form of $\Delta$ in the fppf topology; but the automorphism group scheme of $\Delta$ is constant, so this form is in fact a form in the étale topology, and it is trivial.

The class of the gerbe $\mathcal{M}$ banded by $\Delta$ is classified by the cohomology group $\mathrm{H}_{\mathrm{fppf}}^{2}(\operatorname{Spec} k, \Delta)$. 
Lemma 3.12. If $\Delta$ is a diagonalizable group scheme over a separably closed field $k$, we have $\mathrm{H}_{\mathrm{fppf}}^{2}(\operatorname{Spec} k, \Delta)=0$.

Proof. The group scheme $\Delta$ is a product of groups of the form $\boldsymbol{\mu}_{n}$, so it is enough to consider the case $\Delta=\boldsymbol{\mu}_{n}$. Then the result follows from the Kummer exact sequence of fppf sheaves

$$
0 \longrightarrow \boldsymbol{\mu}_{n} \longrightarrow \mathbb{G}_{\mathrm{m}} \stackrel{\times n}{\longrightarrow} \mathbb{G}_{\mathrm{m}} \longrightarrow 0
$$

and the fact that $\mathrm{H}_{\mathrm{fppf}}^{i}\left(\operatorname{Spec} k, \mathbb{G}_{\mathrm{m}}\right)=\mathrm{H}_{\text {ét }}^{i}\left(\operatorname{Spec} k, \mathbb{G}_{\mathrm{m}}\right)=0$ for $i>0$ (see [21, III, Theorem 3.9]).

This concludes the proofs of Propositions 3.7 and 3.6 and of Theorem 3.2 .

\section{Appendix A. RigidificAtion}

In this section we discuss the notion of rigidification, where a subgroup $G$ of inertia is "removed". This was studied in [2, 28, 5] when $G$ is in the center of inertia, the general case briefly mentioned in [17.

In what follows, when we refer to a sheaf on a scheme $T$ this will be a sheaf on the fppf site of $T$.

Let $S$ be a scheme, or an algebraic space, and let $\mathcal{X} \rightarrow S$ be a locally finitely presented algebraic stack. We will denote by $\mathcal{I} \mathcal{X} \rightarrow \mathcal{X}$ the inertia stack.

Suppose that $G \subseteq \mathcal{I X}$ is a flat finitely presented subgroup stack. This means that it is closed substack, the neutral element section $\mathcal{X} \rightarrow \mathcal{I} \mathcal{X}$ factors through $G$, the inverse morphism $\mathcal{I} \mathcal{X} \rightarrow \mathcal{I} \mathcal{X}$ carries $G$ into itself, and the composition morphism $\mathcal{I} \mathcal{X} \times \mathcal{X} \mathcal{I} \mathcal{X} \rightarrow \mathcal{I} \mathcal{X}$ carries $G \times \mathcal{X} G$ into $G$. Let $\xi$ be an object of $\mathcal{X}$ over some $S$-scheme $T$; this corresponds to a morphism $T \rightarrow \mathcal{X}$. The pullback $T \times \mathcal{X} \mathcal{I} \mathcal{X}$ is, canonically, the group scheme $\underline{\mathrm{Aut}}_{T}(\xi) ;$ the pullback of $G$ to $T$ gives a flat group subscheme $G_{\xi} \subseteq \underline{A u t}_{T}(\xi)$. If $\xi \rightarrow \xi^{\prime}$ is an arrow in $\mathcal{X}$ mapping a morphism of $S$-schemes $T \rightarrow T^{\prime}$, we have a canonical isomorphism $\underline{\mathrm{Aut}}_{T^{\prime}}\left(\xi^{\prime}\right) \simeq T^{\prime} \times_{T} \underline{\mathrm{Aut}}_{T}(\xi)$; this restricts to an isomorphism $G_{\xi^{\prime}} \simeq T^{\prime} \times_{T} G_{\xi}$.

Conversely, assume that for each object $\xi$ of $\mathcal{X}$ we have a subgroup scheme $G_{\xi} \subseteq \underline{\operatorname{Aut}}_{T}(\xi)$ which is flat and finitely presented over $T$, such that for each arrow $\xi \rightarrow \xi^{\prime}$ mapping to a morphism $T \rightarrow T^{\prime}$ the canonical isomorphism Aut $_{T^{\prime}}\left(\xi^{\prime}\right) \simeq T^{\prime} \times_{T} \underline{\operatorname{Aut}}_{T}(\xi)$ carries $G_{\xi^{\prime}}$ isomorphically onto $T^{\prime} \times_{T} G_{\xi}$. Then there is a unique flat finitely presented subgroup stack $G \subseteq \mathcal{I} \mathcal{X}$ such that for any object $\xi$ of $\mathcal{X}(T)$ the pullback of $G$ to $T$ coincides with $G_{\xi}$.

Notice that this condition implies that each $G_{\xi}$ is normal in $\operatorname{Aut}_{T}(\xi)$. In fact, if $\xi$ is an object of $\mathcal{X}(T)$ and $u$ is in $\operatorname{Aut}_{T}(\xi)$, the automorphism of $\underline{\mathrm{Aut}}_{T}(\xi)$ induced by the arrow $u: \xi \rightarrow \xi$ is conjugation by $u$, and carries $G_{\xi}$ into itself.

Theorem A.1. There exists a locally finitely presented algebraic stack $\mathcal{X} \rrbracket G$ over $S$ with a morphism $\rho: \mathcal{X} \rightarrow \mathcal{X} \rrbracket G$ with the following properties. 
(a) $\mathcal{X}$ is an fppf gerbe over $\mathcal{X} \rrbracket G$.

(b) For each object $\xi$ of $\mathcal{X}(T)$, the homomorphism of group schemes

$$
\underline{\operatorname{Aut}}_{T}(\xi) \longrightarrow \underline{\operatorname{Aut}}_{T}(\rho(\xi))
$$

is surjective with kernel $G_{\xi}$.

Furthermore, if $G$ is finite over $\mathcal{X}$ then $\rho$ is proper; while if $G$ is étale then $\rho$ is also étale.

Remark A.2. It is not hard to show that these properties characterize $\mathcal{X} \rrbracket G$ uniquely up to equivalence.

Furthermore, the sheaf of groups $G$ on $\mathcal{X}$ descends to a band $\underline{G}$ over $\mathcal{X} \rrbracket G$, and $\mathcal{X}$ is banded by $\underline{G}$ over $\mathcal{X} \rrbracket G$. Using this one can give a modular interpretation of $\mathcal{X} \rrbracket G$ in a spirit similar to that of [3, Proposition C.2.1].

Example A.3. Suppose that $S$ is a locally noetherian scheme, $\mathcal{X} \rightarrow S$ a regular Deligne-Mumford stack with finite inertia, locally of finite type over $S$. We can take as $G$ the closure of the fibers of $\mathcal{I} \mathcal{X}$ over the generic points of the irreducible components of the moduli space of $\mathcal{X}$. The morphism $G \rightarrow \mathcal{X}$ is easily seen to be étale; hence we can construct a rigidification $\mathcal{X} \square G$, which is a regular Deligne-Mumford stack with trivial generic stabilizers. Therefore every regular Deligne-Mumford is an étale gerbe over a regular Deligne-Mumford stack with trivial generic stabilizers.

This fact was used, for example, in [18, without adequate justification.

Proof. Let us define a fibered category $\overline{\mathcal{X}}$ over $S$ in the following fashion.

The objects of $\overline{\mathcal{X}}$ are the objects of $\mathcal{X}$.

Let $f: T \rightarrow T^{\prime}$ be a morphism of $S$-schemes, $\xi$ and $\xi^{\prime}$ be objects of $\mathcal{X}$ mapping to $T$ and $T^{\prime}$ respectively. The set of arrows $\xi \rightarrow \xi^{\prime}$ mapping to $f$ is in a canonical bijective correspondence with the global sections of the sheaf $\underline{\operatorname{Isom}}_{T}\left(\xi, f^{*} \xi^{\prime}\right)$. There is a faithful right action of $G_{\xi}$ on the algebraic space $\underline{\operatorname{Isom}}_{T}\left(\xi, f^{*} \xi^{\prime}\right)$

Notice the following fact. There is also a left action of $T \times_{T^{\prime}} G_{\xi^{\prime}}$ on $\underline{\operatorname{Isom}}_{T}\left(\xi, f^{*} \xi^{\prime}\right)$ by composition. This commutes with the action of $G_{\xi}$. Furthermore, it is easy to see that the induced action of $T \times_{T^{\prime}} G_{\xi^{\prime}}$ on the quotient $\underline{\operatorname{Isom}}_{T}\left(\xi, f^{*} \xi^{\prime}\right) / G_{\xi}$ is trivial; hence the quotient $\underline{\operatorname{Isom}}_{T}\left(\xi, f^{*} \xi^{\prime}\right) / G_{\xi}$ coincides with the double quotient $G_{\xi^{\prime}} \backslash \underline{\operatorname{Isom}}_{T}\left(\xi, f^{*} \xi^{\prime}\right) / G_{\xi}$.

We define an arrow $\xi \rightarrow \xi^{\prime}$ in $\overline{\mathcal{X}}$ mapping to $f$ as the set of global sections of the quotient sheaf $\underline{\operatorname{Isom}}_{T}\left(\xi, f^{*} \xi^{\prime}\right) / G_{\xi}$.

Let $g: T^{\prime} \rightarrow T^{\prime \prime}$ be a morphism of $S$-schemes, and let $\xi^{\prime \prime}$ be an object of $\mathcal{X}$ mapping to $T^{\prime \prime}$. Composition defines a morphism of algebraic spaces

$$
\underline{\operatorname{Isom}}_{T}\left(\xi, f^{*} \xi^{\prime}\right) \times_{T^{\prime}} \underline{\operatorname{Isom}}_{T^{\prime}}\left(\xi^{\prime}, g^{*} \xi^{\prime \prime}\right) \longrightarrow \underline{\operatorname{Isom}}_{T}\left(\xi,(g f)^{*} \xi^{\prime \prime}\right)
$$

which descends to a morphism

$$
\underline{\operatorname{Isom}}_{T}\left(\xi, f^{*} \xi^{\prime}\right) / G_{\xi} \times_{T^{\prime}} \underline{\operatorname{Isom}}_{T^{\prime}}\left(\xi^{\prime}, g^{*} \xi^{\prime \prime}\right) / G_{\xi^{\prime}} \longrightarrow \underline{\operatorname{Isom}}_{T}\left(\xi,(g f)^{*} \xi^{\prime \prime}\right) / G_{\xi} ;
$$

this defines the composition in $\overline{\mathcal{X}}$. 
The fibered category $\overline{\mathcal{X}}$ is a prestack, but not a stack. We define $\mathcal{X} \rrbracket G$ to be the stack over $S$ associated with $\overline{\mathcal{X}}$. The morphism $\rho: \mathcal{X} \rightarrow \mathcal{X} \rrbracket G$ is the composite of the two obvious morphisms $\mathcal{X} \rightarrow \overline{\mathcal{X}}$ and $\overline{\mathcal{X}} \rightarrow \mathcal{X} \rrbracket G$.

Let $T$ be and $S$-scheme, $\bar{\xi}$ and $\bar{\eta}$ two objects in $(\mathcal{X} \rrbracket G)(T)$. We claim that the sheaf $\operatorname{Isom}_{T}(\bar{\xi}, \bar{\eta})$ is an algebraic space. This is a local problem in the fppf topology, by a result of Artin ([20, Corollaire 10.4.1]), so we may assume that $\bar{\xi}$ and $\bar{\eta}$ are the images of two objects $\xi$ and $\eta$ of $\mathcal{X}(T)$. In this case $\operatorname{Isom}_{T}(\bar{\xi}, \bar{\eta})$ coincides with the quotient $\underline{\operatorname{Isom}}_{T}\left(\xi, f^{*} \xi^{\prime}\right) / G_{\xi}$, which is an algebraic space, again by Artin's result ([20, Corollaire 10.4]).

Now we check that the morphism $\rho: \mathcal{X} \rightarrow \mathcal{X} \rrbracket G$ is represented by smooth algebraic stacks which are fppf gerbe. More precisely, we now show that, given a morphism $T \rightarrow \mathcal{X} \rrbracket G$, where $T$ is a scheme, the pullback $T \times{ }_{\mathcal{X} / G} \mathcal{X}$ is fppf locally isomorphic to a stack of the form $\mathcal{B}_{T} G_{\xi}$. This implies the statement of the theorem. If $U \rightarrow \mathcal{X}$ is a smooth morphism, then the composition $U \rightarrow \mathcal{X} \rrbracket G$ is also smooth, which shows that $\mathcal{X} \rrbracket G$ is an algebraic stack. Furthermore $\mathcal{B}_{T} G_{\xi}$ is proper over $T$ when $G_{\xi}$ is finite, and étale when $G_{\xi}$ is étale.

We may assume that the object $\bar{\xi}$ of $(\mathcal{X} \emptyset G)(T)$ corresponding to the morphism $T \rightarrow \mathcal{X} \rrbracket G$ comes from an object $\xi$ of $\mathcal{X}(T)$. An object of the fiber product $T \times \mathcal{X} \mathbb{G}$ X consist of the following data:

(a) A morphism of schemes $f: T^{\prime} \rightarrow T$;

(b) An object $\eta$ of $\mathcal{X}\left(T^{\prime}\right)$;

(c) A section

$$
T^{\prime} \longrightarrow \underline{\operatorname{Isom}}_{T^{\prime}}(\rho(h), \bar{\xi})=\underline{\operatorname{Isom}}_{T^{\prime}}(\eta, \xi) / G_{\xi} .
$$

From this data we obtain a $G_{\xi}$-torsor $P \rightarrow T^{\prime}$, where

$$
P \stackrel{\text { def }}{=} T^{\prime} \times_{\underline{\operatorname{Isom}}_{T^{\prime}}(\eta, \xi) / G_{\xi}} \underline{\operatorname{Isom}}_{T^{\prime}}(\eta, \xi) .
$$

By examining what happens to arrows it is easy to show that this construc-

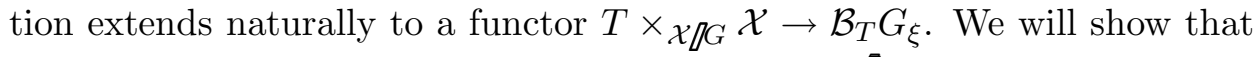
this is an equivalence. The inverse $\mathcal{B}_{T} G_{\xi} \rightarrow T \rightarrow \mathcal{X} \rrbracket G$ is defined as follows. Let $T^{\prime}$ be a scheme over $T$ and $\phi: P \rightarrow T^{\prime}$ be a $G_{\xi}$-torsor. The action of $G_{\xi}$ on $\xi$ gives to an action of $G_{\xi}$ on the pullback $\xi_{T}$; by [32, Theorem 4.46] we obtain an object $\eta$ of $\mathcal{X}\left(T^{\prime}\right)$. The functor $\mathcal{B}_{T} G_{\xi} \rightarrow T \rightarrow \mathcal{X} \rrbracket G$ sends $P \rightarrow T^{\prime}$ into $\eta$. We leave it to the reader to check that this gives an inverse to the functor above. This concludes the proof.

\section{REFERENCES}

1. D. Abramovich and A. Vistoli, Compactifying the space of stable maps, J. Amer. Math. Soc 15 (2002), 27-75.

2. D. Abramovich, A. Corti, and A. Vistoli, Twisted bundles and admissible covers, Comm. Algebra 31 (2003), 3547-3618.

3. D. Abramovich, T. Graber and A. Vistoli, Gromov-Witten theory of DeligneMumford stacks, preprint, http://www.arxiv.org/abs/math.AG/0603151 
4. M. Artin, Versal deformations and algebraic stacks, Invent. Math. 27 (1974), 165189.

5. K. Behrend and B. Noohi, Uniformization of Deligne-Mumford curves, J. Reine Angew. Math., to appear.

6. P. Berthelot, A. Grothendieck, and L. Illusie, Théorie des Intersections et Théorème de Riemann-Roch (SGA 6), Springer Lecture Notes in Math 225 (1971).

7. B. Conrad, Keel-Mori theorem via stacks, preprint available at http://www.math.1sa.umich.edu/ bdconrad

8. P. Deligne and D. Mumford, The irreducibility of the space of curves of given genus, Inst. Hautes Études Sci. Publ. Math. 36 (1969), 75-109.

9. M. Demazure et al., Schémas en groupes, Lecture Notes in Mathematics 151, 152 and 153, Springer-Verlag (1970).

10. J. Dieudonné and A. Grothendieck, Éléments de géométrie algébrique, Inst. Hautes Études Sci. Publ. Math. 4, 8, 11, 17, 20, 24, 28, 32 (1961-1967).

11. D. Gorenstein, Finite groups. Chelsea Publishing Co., New York, 1980. xvii+519 pp.

12. Jean Giraud, Cohomologie non abélienne, Springer-Verlag, Berlin, 1971, Die Grundlehren der mathematischen Wissenschaften, Band 179.

13. L. Gruson and M. Raynaud, Critères de platitude et de projectivité. Techniques de "platification" d'un module, Invent. Math. 13 (1971), 1-89.

14. L. Illusie, Complexe cotangent et déformations. I, Lecture Notes in Mathematics, Vol. 239 Springer, Berlin, 1971.

15. N. Jacobson, Lie algebras. Republication of the 1962 original. Dover Publications, Inc., New York, 1979. ix+331 pp. ISBN: 0-486-63832-4

16. S. Keel and S. Mori, Quotients by groupoids, Ann. of Math. (2) 145 (1997), no. 1, 193-213.

17. A. Kresch, Geometry of Deligne-Mumford stacks, preprint.

18. A. Kresch, A. Vistoli: On coverings of Deligne-Mumford stacks and surjectivity of the Brauer map. Bull. London Math. Soc. 36 (2004), 188-192.

19. S. Kleiman, The Picard scheme, preprint (2005).

20. G. Laumon and L. Moret-Bailly, Champs Algébriques. Ergebnisse der Mathematik un ihrer Grenzgebiete 39, Springer-Verlag, 2000.

21. J.S. Milne, Étale cohomology, Princeton University Press (1980).

22. D. Mumford Abelian varieties. Tata Institute of Fundamental Research Studies in Mathematics, No. 5. Published for the Tata Institute of Fundamental Research, Bombay; Oxford University Press, London 1970 viii+242 pp.

23. M. Olsson, Sheaves on Artin stacks, J. Reine Angew. Math., to appear. http://www.math.berkeley.edu/ molsson/qcohrevised.pdf

24. - Hom-stacks and restriction of scalars, to appear in Duke Math. J.

25. - On proper coverings of Artin stacks, Advances in Mathematics 198 (2005), 93-106.

26. Deformation theory of representable morphisms of algebraic stacks, to appear in Math. Zeit.

27. - A boundedness theorem for Hom-stacks, preprint 2005.

28. M. Romagny, Group actions on stacks and applications. Michigan Math. J. 53 (2005), no. 1, 209-236.

29. N. Saavedra Rivano, Catégories Tannakiennes. Lecture Notes in Mathematics, Vol. 265. Springer-Verlag, Berlin-New York, 1972.

30. N. Saavedra Rivano, Catégories Tannakiennes. Lecture Notes in Mathematics, Vol. 265. Springer-Verlag, Berlin-New York, 1972.

31. R. W. Thomason, Algebraic K-theory of group scheme actions, in Algebraic Topology and Algebraic K-theory, Annals of Mathematical Studies 113, Princeton University Press, Princeton (1987) 
32. A. Vistoli, Grothendieck topologies, fibered categories and descent theory, in Fundamental algebraic geometry, 1-104, Math. Surveys Monogr., 123, Amer. Math. Soc., Providence, RI, 2005.

(Abramovich) Department of Mathematics, Brown University, Box 1917, ProvIDENCE, RI 02912, U.S.A.

E-mail address: abrmovic@math.brown.edu

(Olsson) Department of Mathematics \#3840, University of California, BerkeLEY, CA 94720-3840, U.S.A.

E-mail address: molsson@math.berkeley.edu

(Vistoli) Scuola Normale Superiore, Piazza dei Cavalieri 7, 56126 Pisa, Italy

E-mail address: angelo.vistoli@sns.it 